
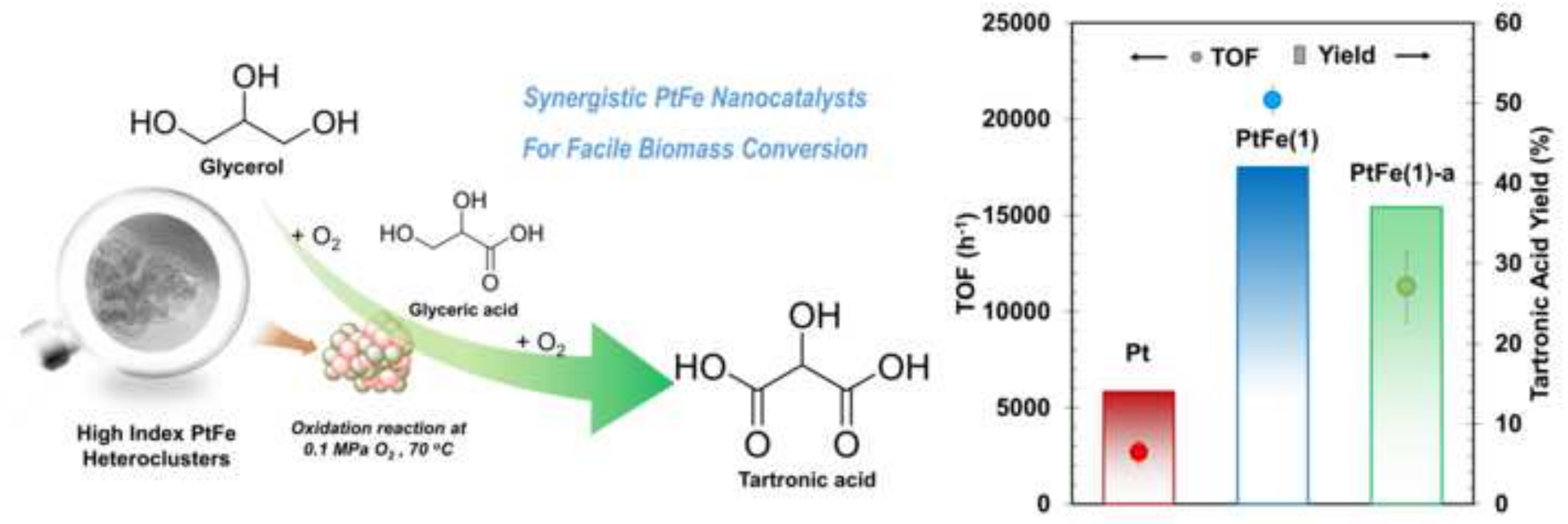


\section{Anisotropic Growth of PtFe Nanoclusters Induced by Lattice- Mismatch: Efficient Catalysts for Oxidation of Biopolyols to Carboxylic Acid Derivatives}

Xin Jin,${ }^{1 \$}$ Meng Zhao, ${ }^{1 \$}$ Wenjuan Yan,,${ }^{1,2}$ Chun Zeng, ${ }^{1}$ Pallavi Bobba, ${ }^{1,2}$ Prem S. Thapa, ${ }^{3}$ Bala Subramaniam, ${ }^{1,2}$ Raghunath V. Chaudhari ${ }^{1,2^{*}}$

${ }^{1}$ Center for Environmentally Beneficial Catalysis, University of Kansas, 1501 Wakarusa Drive, Lawrence, Kansas 66047, USA

${ }^{2}$ Department of Chemical and Petroleum Engineering, University of Kansas, $1530 \mathrm{~W} 15^{\text {th }}$ St., Lawrence, Kansas 66045, USA

${ }^{3}$ Microscopy and Analytical Imaging Laboratory, Haworth Hall, 1200 Sunnyside Ave, University of Kansas, Lawrence, Kansas 66045, USA

${ }^{\$}$ Equally contributing authors

"Corresponding author: rvc1948@ku.edu 


\begin{abstract}
By exploiting the large difference in lattice constants between $\mathrm{Pt}$ and Fe metals, anisotropic growth of bimetallic PtFe clusters with high index numbers have been prepared to investigate their catalytic performance in oxidation of biopolyols. Surface characterization using transmission electron microscopy shows that etching effects caused by $\mathrm{Fe}^{3+}$ and anisotropic growth induced by lattice mismatch contributed to the final nanocrystal geometry. The bimetallic PtFe heterocluster is shown to exhibit an unprecedented six-fold enhanced catalytic activity and three-fold higher selectivity compared to monometallic Pt and Fe catalysts, in oxidation of biomass-derived polyols to dicarboxylic acids. A detailed study on structure-dependent kinetics of oxidation of glycerol, based on concentration-time profiles on $\mathrm{Pt}$ and PtFe catalysts, reveals that the presence of Fe in Pt catalysts significantly enhances the rate of oxidation and decreases the activation barriers for primary and secondary oxidation steps leading to enhanced catalytic activity and selectivity. The lattice mismatch methodology employed in this work provides a unique tool for designing high index catalytically active materials for various other industrial applications.
\end{abstract}

Key words: Lattice mismatch, oxidation, catalysis, biomass conversion, glycerol 


\section{Introduction}

The development of novel materials with enhanced functionalities such as magnetics, plasmonics and catalysis is a key to solving current energy and environmental issues.[1,2] Catalytic conversion of sustainable feedstocks to value-added chemicals provides fundamental building blocks for making everyday products.[3, 4] In addition to significant improvements in activity/selectivity and stability, one of the major goals in developing cost-effective catalysts is to reduce the use of noble metals by incorporating inexpensive and earth-abundant replacements. Anisotropic bimetallic nanoparticles with tunable active sites and metal-metal interactions have shown promising performances in magnetics and plasmonics,[5] but the benefits of novel bimetallic structures have not been fully exploited for catalytic applications. At present, a two-step seeded growth approach is used to force the formation of anisotropic nanoparticles.[6, 7] Selfarrangement of two different metal atoms in an anisotropic fashion is difficult as most metals tend to display stable face-centered-cubic $(f c c)$ structures.[8] By using PtFe bimetallic nanocrystal as an example, we report here a unique synthesis of solventmediated self-assembly of anisotropic nanoclusters, induced by lattice mismatch, to achieve novel bimetallic structures in one pot. More importantly, it is demonstrated that the bimetallic PtFe nanoclusters exhibit remarkable catalytic performances for low temperature oxidation of renewable biomass derived feedstocks to value-added chemicals.

Given that fossil-based resources are not sustainable, there has been an intense search globally for alternative feedstocks to make chemical intermediates. In this regard, the catalytic synthesis of petrochemical equivalents from renewable biomass feedstocks has 
received significant interest in recent decades.[9] For example, aqueous phase oxidation (APO) of biomass provides a promising route for manufacturing a variety of products, such as intermediates for renewable polymers, nontoxic anti-freeze agents, BPA-free plastics and environmentally degradable detergents. Hydroxy dicarboxylic acids (HDCAs, such as tartronic acid, glucaric acid, and oxalic acid), derived from the oxidation of sugars and polyols, have high bio-activity and degradability in natural environments.[1012] They are regarded as some of the most promising intermediates in polymer and pharmaceutical industries, and are currently manufactured from petrochemical feedstocks using corrosive/toxic reagents and severe operating conditions.[13-15] Among the challenges in HDCA synthesis are the difficulties in achieving high selectivity, elimination of stoichiometric reagents and avoidance of waste inorganic products. The known routes for making them involve the use of either toxic and corrosive mineral chemicals (e.g. nitric acid, bleaches) as oxidants or expensive noble metal catalysts in the presence of molecular oxygen.[14] While the latter route possesses greener characteristics, it suffers from several major technical challenges including poor catalyst activity and selectivity to HDCAs.[14, 16, 17] Consider glycerol, an abundantly available sugar polyol from biomass feedstocks, as a representative example (Figure 1).[18] Primary oxidation of glycerol to glyceric acid, a monocarboxylic acid, is easily achieved in the presence of various metal catalysts. However, further (secondary) oxidation of glyceric acid to more valuable tartronic acid, is extremely difficult due to the poor oxidation activity. Several mono and bimetallic catalyst systems such as Pd, Pt and Au have been extensively studied on glycerol oxidation. But most of the experimental results show that glyceric acid is the major product while selectivity towards tartronic 
acid is low. In particular, PdAu is the most intensively studied system.[19] The effects of reaction temperature, $\mathrm{O}_{2}$ pressure, $\mathrm{pH}$ values as well as metal particle sizes, metal compositions, solid support have been the main focus in previous studies and they are found to influence the catalyst activity (T: $60-100{ }^{\circ} \mathrm{C}$, TOF: $500-21,000 \mathrm{~h}^{-1}$ ) in aqueous phase glycerol oxidation.[20, 21] In addition, bimetallic PdAg, PdPt and PdSn catalysts were also studied but tartronic acid selectivity is also very low (S: 0-18\%).[22, 23] Aubased catalysts supported on carbon is found to exhibit excellent oxidation activity (T: 25-120 ${ }^{\circ} \mathrm{C}$, TOF: 1774 $\mathrm{h}^{-1}$ ) but poor selectivity towards tartronic acid (S: 3-12\%).[24] The best results reported so far in literature, are (a) Au/HY catalyst (TOF: $40 \mathrm{~h}^{-1}, \mathrm{~S}$ : 82\%),[25] (b) PdBi/C catalyst (TOF: $0.96 \mathrm{~h}^{-1}, \mathrm{~S}: 93 \%$ ) with poor activity[26] and (c) $\mathrm{PtCe} / \mathrm{C}$ catalyst (TOF $\left.<1 \mathrm{~h}^{-1}, \mathrm{~S}: 38 \%\right)$.[27]

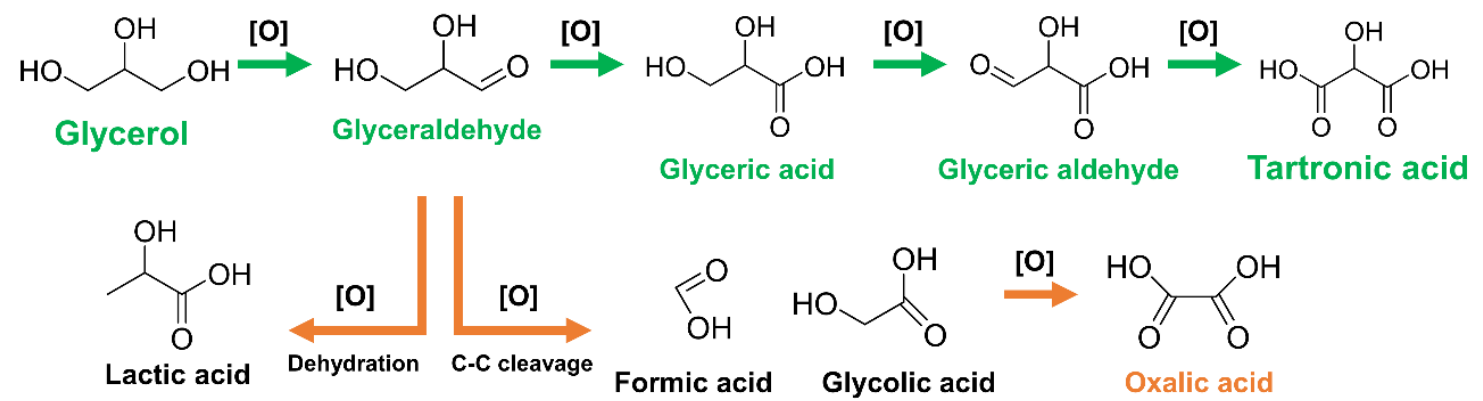

Figure 1. Oxidation of biomass derived glycerol to value-added carboxylic acids

Pt-based materials are generally regarded as the most promising oxidation catalysts.[11, 14, 28-31] There have been several attempts to develop bimetallic $\mathrm{Pt}_{\mathrm{x}} \mathrm{M}$ (x: 0.5-7, M: Cu, $\mathrm{Ni}, \mathrm{Co}, \mathrm{Au}, \mathrm{Pd})[2,6,32,33]$ catalysts to explore a possible enhancement in their catalytic oxidation activity and selectivity. For the preparation of Pt-based bimetallic crystals, a two-pot template growth is the known synthetic strategy, wherein anisotropic foreign species are forced to assemble on pre-existing seeds, to obtain well-defined nanocubes, 
rhombics, tetrahedral, concave and star structures.[33-36] The lattice mismatch between template species and shell materials is the main driving force for anisotropic growth. Even though several of these bimetallic materials have been studied in glycerol oxidation, only limited enhancement if any has been reported in terms of improved catalytic performance (TOF: $270-4,200 \mathrm{~h}^{-1}$ ). This is because these well-defined $\mathrm{Pt}_{\mathrm{x}} \mathrm{M}$ nanocrystals often possess the low index [111] and [100] surfaces, which are not ideal for oxygen activation. $[10,12,37,38]$ Consequently, these materials often show low catalytic activity in glycerol oxidation and easily cause significant side reactions such as $\mathrm{C}-\mathrm{C}$ cleavage, decarbonylation and decarboxylation generating $\mathrm{CO}_{2}$ and less valued carbonate salts (selectivity: 5-27\%) at moderately elevated oxidation temperatures (T: $\left.90-160{ }^{\circ} \mathrm{C}\right) .[12$, $29,39-43$ ] Poor selectivity towards valuable HDCA products ( $\mathrm{S}<15 \%$ ) is thus the major issue plaguing current glycerol oxidation catalysts.

High price of noble metals but poor oxidation performances motivated us to design more active and cost effective catalytic materials for selective HDCA synthesis. In this paper, we report a simple solvent-mediated method for self-assembly of anisotropic nanoclusters. By using $\mathrm{Pt}$ and $\mathrm{Fe}$ as an example, we take advantage of lattice constant mismatch between the two metals (Pt: $0.392 \mathrm{~nm}, \mathrm{Fe}: 0.287 \mathrm{~nm} ; 27 \%$ mismatch)[44] to achieve anisotropic growth of PtFe crystals and simultaneous immobilization on solid catalyst supports in one pot. There have been several previous studies to understand structural isotropy and anisotropy of bimetallic PtFe nanocrystals. Most research reports have focused primarily on the influence of structural changes on magnetic characteristics. Up to date, fundamentals on lattice mismatched PtFe crystal growth and its surface catalytic activity in liquid phase oxidation of biomass derived molecules are still unknown. In 
order to better understand the influence of lattice mismatched PtFe nanoclusters on catalytic activity and selectivity, we chose to systematically investigate the oxidation of glycerol, at relatively mild conditions $\left(60-80{ }^{\circ} \mathrm{C}, 0.1 \mathrm{MPa} \mathrm{O}_{2}\right)$ as a case study. We report for the first time herein that anisotropic PtFe clusters exhibit a remarkable six-fold enhancement in the oxidation activity and three-fold higher selectivity to HDCAs compared with monometallic Pt and Fe catalysts. An unprecedented production rate of $227.6 \mathrm{~h}^{-1}$ for tartronic acid (S: 42\%) formation and 20,978 $\mathrm{h}^{-1}$ in TOF are achieved. Kinetic study was used as a tool to reveal the role of Fe species in Pt catalysts during oxidation reactions. Detailed concentration-time profiles at various temperatures were obtained to understand the relation between surface structure of the catalyst and the kinetics of oxidation. The lattice mismatch methodology for synthesis of novel bimetallic PtFe catalysts and the specific case study shown here are expected to have wider impacts on designing high performance catalytic materials for highly rewarding applications such as the oxidative synthesis of chemicals and fuels from plant-based biomass.

\section{Materials and Methods}

2.1 Chemicals. All chemicals used in this paper were purchased from Sigma Aldrich. $\mathrm{CeO}_{2}$ (nanopowder, < $\left.25 \mathrm{~nm}\right), \operatorname{Pt}(\mathrm{acac})_{2}(97 \%) \mathrm{Fe}(\mathrm{acac})_{3}(97 \%)$, dimethylformamide (DMF, 99.8\%), ethanol (anhydrous), glycerol (99.5\%), glyceric acid (70 wt\% in $\left.\mathrm{H}_{2} \mathrm{O}\right)$, tartronic acid (97\%), lactic acid (98\%), glycolic acid (97\%), oxalic acid (98\%), formic acid (95\%), $\mathrm{H}_{2} \mathrm{SO}_{4}(95-98 \%), \mathrm{NaOH}(97 \%), \mathrm{N}_{2}$ (high purity), $\mathrm{O}_{2}$ (high purity).

2.2 Synthesis of monometallic Pt and Fe catalysts supported on $\mathrm{CeO}_{2}$. We used a solvothermal synthesis method to prepared monometallic Pt and Fe nanocatalysts. Specifically, about $20 \mathrm{mM}$ of $\mathrm{Pt}(\mathrm{acac})_{2}$ or $\mathrm{Fe}(\mathrm{acac})_{3}$ were mixed with $20 \mathrm{~mL}$ of $\mathrm{DMF}$ and 
certain amounts of $\mathrm{CeO}_{2}$ powders in a glass insert. The mixture was then transferred to a $100 \mathrm{~mL}$ Parr reactor. The reactor was sealed and flushed with $\mathrm{N}_{2}$ thrice. The reactor was charged with 10 bar $\mathrm{N}_{2}$ pressure at room temperature, before heating to target synthesis temperature $\left(e . g .200^{\circ} \mathrm{C}\right)$. The slurry was stirred at $800 \mathrm{RPM}$ for $12 \mathrm{~h}$ and then cooled down to room temperature. The solid catalyst samples were centrifuged and washed with ethanol/ $\mathrm{H}_{2} \mathrm{O}$ mixture $(2 / 1 \mathrm{vol} / \mathrm{vol})$ at least seven times to remove the surface DMF residues during catalyst synthesis. The as prepared catalysts were dried in a vacuum oven at $60{ }^{\circ} \mathrm{C}$ overnight before using in oxidation tests.

2.3 Synthesis of bimetallic PtFe catalysts supported on $\mathbf{C e O}_{2}$. The procedures are similar to these shown in preparing monometallic catalysts. Specifically, $20 \mathrm{mM}$ of $\operatorname{Pt}(\mathrm{acac})_{2}$ with different amounts $\mathrm{Fe}(\mathrm{acac})_{2}$ were mixed with $\mathrm{DMF}$ and predetermined amounts of $\mathrm{CeO}_{2}$ powder in a glass insert. The molar ratio of $\mathrm{Pt} / \mathrm{Fe}$ was varied from 1/0.5, 1/1, 1/2. The catalysts were denoted as $\operatorname{PtFe}(\mathbf{0 . 5}), \operatorname{PtFe}(\mathbf{1}), \operatorname{PtFe}(2)$, respectively. $\operatorname{PtFe}(1)$ sample was annealed under $\mathrm{H}_{2}$ atmosphere. Specifically, the sample was first flushed with $\mathrm{N}_{2}$ in a tubular furnace at room temperature, after which it was heated to $150{ }^{\circ} \mathrm{C}$. Then the gas was switched to $\mathrm{H}_{2}$ and the furnace was further heated to $400{ }^{\circ} \mathrm{C}$. The annealed sample is denoted as $\mathrm{PtFe}(1)$-a. In all mono and bimetallic catalysts, the weight loading of Pt is $1 \mathrm{w} \%$ (confirmed by ICP).

2.4 Surface Characterization. Transmission electron microscopy (TEM): sample preparation and detailed procedures were similar to that previously described.[18] Samples were prepared by mixing solid catalyst with ethanol and agitating in an ultrasonic bath. $10 \mu \mathrm{L}$ of catalyst-containing sample was placed onto a copper mesh grid. The wet grid was allowed to air-dry for several minutes prior to examination under 
TEM.[45] Around 200 particles were measured and average particle size as well as standard deviation were calculated. Particle size measurement (from 150 clusters/particles) of each sample was carried out using ImageJ software. This information is used to calculate numbers of surface active site of Pt catalysts. Other surface properties including catalyst surface areas, pore volumes, metal dispersion and compositions are presented in Table S1. X-ray photoelectron spectroscopy (XPS) characterization and metal leaching were carried out by Evans Analytical Group (www.eag.com, see supporting information for detailed information).

2.5 Oxidation of glycerol. The experimental procedure for testing catalyst activity for glycerol oxidation was similar to that described previously.[14] Briefly, about $0.05 \mathrm{~g}$ of solid catalyst was added to $25 \mathrm{~mL}$ aqueous solution containing glycerol $(1.0 \mathrm{~g})$ and $\mathrm{NaOH}(1.7 \mathrm{~g})$, which was transferred to a $100 \mathrm{~mL}$ of three neck flask. The slurry was heated in an oil bath with precise temperature control before heating up to a predetermined reaction temperature. Once the liquid slurry attained the reaction temperature, stirring rate was set at $1000 \mathrm{RPM}$, and $\mathrm{O}_{2}$ was introduced into the reactor at a constant rate $(60 \mathrm{~mL} / \mathrm{min})$, which signified the start of an experimental run. Small amounts of liquid samples were taken during batch studies and acidified with $\mathrm{H}_{2} \mathrm{SO}_{4}$ solution before injecting in HPLC (SHIMADZU with SH1011 column, see Figure S1 for an example HPLC result). After each batch experiment, the volume of liquid mixture was measured to ensure negligible loss of solvent $(<0.4 \mathrm{~mL})$. From the concentration values, conversion (X), selectivity (S), carbon balance (C\%) and turnover frequency (TOF based on surface Pt atoms, in $\mathrm{h}^{-1}$ ) were calculated as defined below. Due to the complex structures of PtFe bimetallic nanocatalysts, it is difficult to distinguish surface Pt metal 
atoms from Fe. But TOF based on Pt dispersion is still used in this manuscript as an approximation to evaluate the performances of $\mathrm{Pt}$ and $\mathrm{PtFe}$ catalysts in oxidation reactions.

Conversion $=\frac{C_{\text {moless,glycerol }}^{\text {initial }}-C_{\text {moles, }, \text { glycerol }}^{\text {final }}}{C_{\text {moless,glycerol }}^{\text {inity }}}$

Selectivity $=\frac{C_{\text {moles, } \text { products }}^{\text {final }}}{C_{\text {moles, } \text { IIycerol }}^{\text {initial }}-C_{\text {moles, glycerol }}^{\text {final }}}$

$\operatorname{Carbon}(\%)=\frac{C_{\text {moles, } \text { products }}^{\text {final }}}{C_{\text {moles, } \text { colylycerol }}^{\text {corted }}}$

TOF $=\frac{N_{\text {glycerol, converted }}}{N_{\text {surfacePt }} \cdot \text { Time }}($ based on experimental data at conversion $<20 \%)$

For kinetic modeling, pseudo-first order kinetics with regard to glycerol concentration was considered as $\mathrm{O}_{2}$ pressure was kept constant during experiments. Catalyst concentration was the same in all experiments. Both external and intraparticle mass transfer limitations of $\mathrm{O}_{2}$ and glycerol have been evaluated and found to be negligible (see supporting information for calculation and experiment details).[46]

For catalyst reuse experiments, catalyst was separated from reaction mixture by centrifuge at 2000 RPM and washed by deionized water at least three times. Fresh reaction mixture was then mixed with used catalyst immediately and tested for glycerol oxidation without any treatment. The reaction mixtures were stored for leaching tests using ICP. 


\section{Results and Discussion}

\subsection{Catalyst Synthesis, Characterization and Performance Evaluation}

We started the experimental studies by preparing $\mathrm{CeO}_{2}$ supported $\mathrm{Pt}, \mathrm{Fe}$, as well as $\mathrm{PtFe}$ bimetallic crystals with various $\mathrm{Pt} / \mathrm{Fe}$ atomic ratios $(1 / 0.5-1 / 2)$. The specifications of these catalysts are given in supporting information (Table S1). Selected samples were characterized by TEM as shown in Figure 2. Unlike previous reports, where nanocluster growth and immobilization on heterogeneous supports were carried out in successive steps, we synthesized and immobilized Pt based nanocatalysts in DMF solvent under inert $\mathrm{N}_{2}$ atmosphere in one step. We had recently employed this method to prepare lattice matched bimetallic PtCu structures.[14] The TEM images in Figures 2 (a, d) show that the $\mathrm{CeO}_{2}$-immobilized monometallic Pt species are polyhedron nanoparticles with both [111] and [100] surface facets exposed on the support. The average particle size is $11.8 \pm 3.8 \mathrm{~nm}$ (see section 2.4 for details of measurement method). Interestingly, when both $\mathrm{Pt}$ and Fe precursors were present in catalyst synthesis mixture, unique disordered bimetallic PtFe clusters were observed, as manifested in the TEM images [Figures 2 (b, e)]. Due to the large mismatch between the two metals, disordered structures containing both Pt and Fe clusters embedded within each other are dominant in the PtFe(1) sample. As comparing Figures 2 (a, d) with (b, d), we observe that polyhedron shaped particles become disordered heterocluster ones after $\mathrm{Fe}$ species is incorporated (see element scanning in the following section for more information). In addition, the cluster size is found to increase to approximately $17.3 \pm 4.9 \mathrm{~nm}$ after incorporating Fe. To further understand how surface configuration affects catalytic activity of PtFe catalysts, we compared the activity of $\mathrm{PtFe}$ heteroclusters $[\mathrm{PtFe}(1)]$ with ordered layer-by-layer 
[PtFe(1)-a, one Pt atomic layer by one Fe atomic layer] ordered structure. To be more particular, heterocluster structure is defined as segregated $\mathrm{Pt}$ and $\mathrm{Fe}$ domains within one cluster while layer-by-layer one (alloy) has ordered and uniform PtFe layers. Since PtFe crystals are known to undergo phase transformation above Curie temperature, $[47,48]$ the $\mathrm{PtFe}(1)$ sample was annealed under $\mathrm{H}_{2}$ at $400{ }^{\circ} \mathrm{C}$. The annealed sample, designated as PtFe(1)-a, was also characterized by TEM as shown in Figures 2 (c, f). As expected, high index surfaces of $\mathrm{PtFe}(1)$ clusters were transformed into layer-by-layer ordered structures after the annealing process. In other words, the orientation of $\mathrm{PtFe}(1)$-a crystals was completely changed from disordered $f c c$ morphology to highly ordered $L 1_{0}$ structure,[47] without showing any significant changes in cluster sizes $(14.0 \pm 3.9 \mathrm{~nm})$. Detailed images in Figures 2 (e) and (f) confirm the structural changes of a large number of clusters within the $\mathrm{PtFe}(1)$ sample after annealing.

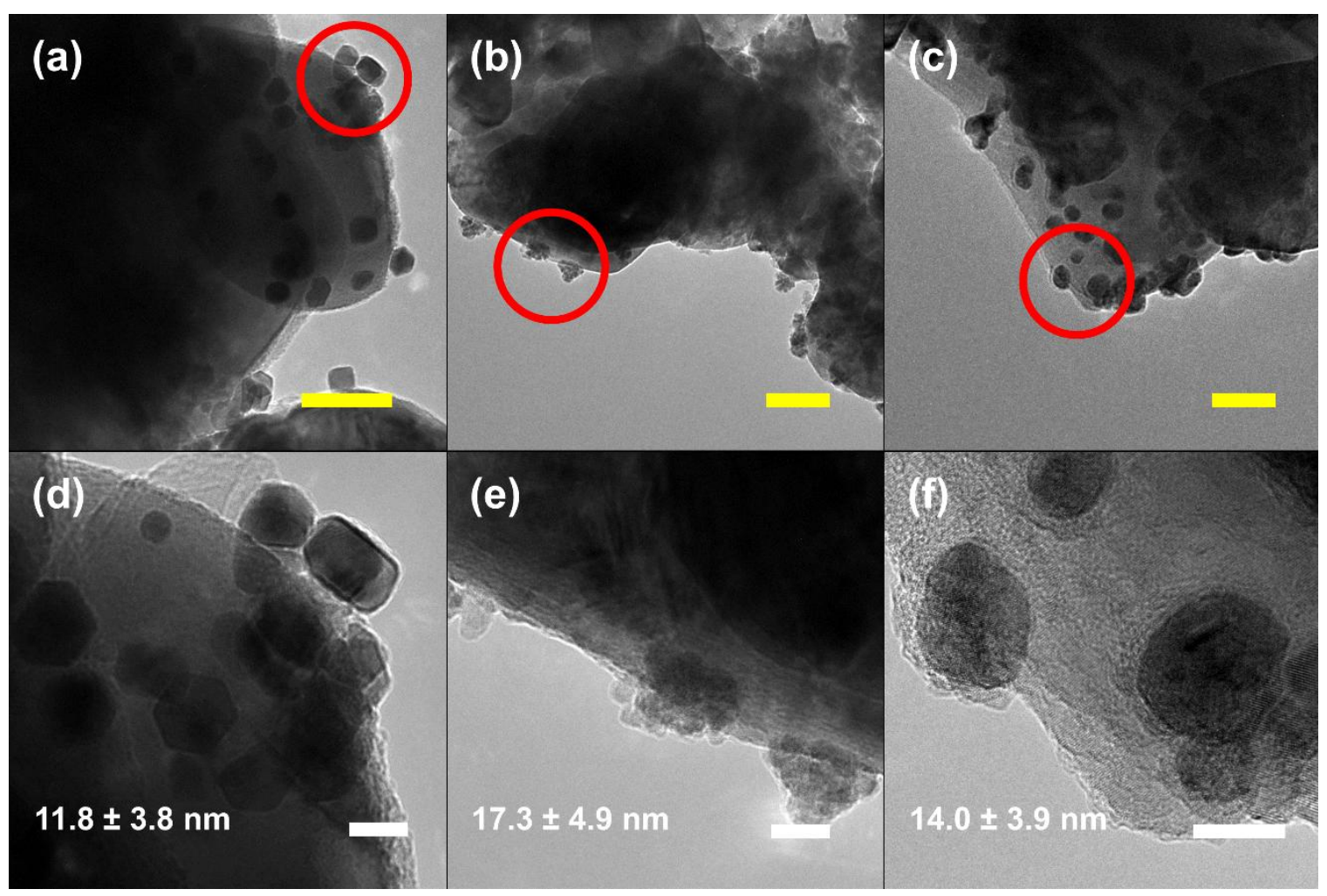


Figure 2. TEM images of $\mathrm{Pt}, \mathrm{PtFe}(1)$ and $\mathrm{PtFe}(1)$-a catalysts supported on $\mathrm{CeO}_{2}$ (yellow and white bars indicate $50 \mathrm{~nm}$ and $10 \mathrm{~nm}$ respectively). (a): Pt, (b): $\mathrm{PtFe}(1)$, (c): $\mathrm{PtFe}(1)-\mathrm{a}$; (d) - (f): HR-TEM images of selected regions in (a) Pt, (b) PtFe(1) and (c) PtFe(1)-a catalysts.

Additional HR-TEM images of Pt, PtFe(1) and PtFe(1)-a samples, which were captured at different sample regions, are shown in Figures 3 (a)-(c). These high resolution images further confirm the unique polyhedron, disordered and layer-by-layer structures of Pt and PtFe clusters on $\mathrm{CeO}_{2}$ support. By measuring the lattice distances, as shown in Figures 3 $(\mathrm{a}, \mathrm{b})$, both Pt and Fe species in heteroclusters can be clearly distinguished.

The distinct catalytic activity of $\mathrm{Pt}, \mathrm{Fe}, \mathrm{PtFe}(1)$ and $\mathrm{PtFe}(1)$-a catalyst samples in the oxidation of glycerol was also investigated to compare the performance of the mono and bimetallic catalysts, the results of which are shown in Figure 3 (d). Clearly, the bimetallic PtFe catalysts show a synergistic enhancement in activity and selectivity for glycerol oxidation. The major oxidation products from glycerol include glyceric, tartronic, lactic, glycolic, oxalic and formic acids.[11] Primary oxidation of glycerol generates glyceraldehyde (GLA) as the key intermediate (Figure 1), which is quickly oxidized to glyceric acid as the major product, that may undergo further (secondary) oxidation of another hydroxyl group to form valuable tartronic acid. If simultaneous dehydration of glycerol occurs, GLA can lead to the formation of lactic acid,[13, 18] while C-C cleavage followed by further oxidation will generate formic and glycolic acids.[10, 28] The catalytic activities were compared for different catalysts based on TOF calculated for $<20 \%$ glycerol conversion and using Pt metal dispersion (\% of surface to bulk Pt content, see Table S1 for details). As shown in Figure 3 (d), monometallic Pt catalyst provides a TOF of 2,677.1 $\pm 656.6 \mathrm{~h}^{-1}$ during oxidation of glycerol. In sharp contrast, bimetallic $\mathrm{PtFe}(1)$ and $\mathrm{PtFe}(1)$-a each display significantly greater TOFs $\left(20,978.2 \pm 707.1 \mathrm{~h}^{-1}\right.$ and 
11,295.1 $\pm 1841.9 \mathrm{~h}^{-1}$, respectively), manifesting remarkable synergistic effects. Moreover, the tartronic acid yield (after $24 \mathrm{~h}$ ) on bimetallic $\mathrm{PtFe}(1)$ and $\mathrm{PtFe}(1)$-a catalysts is about $37-42 \%$, much greater than values observed with monometallic Pt and Fe catalysts (3-14\%). These preliminary results clearly confirm that bimetallic PtFe catalysts display improved catalytic performances compared with monometallic ones.

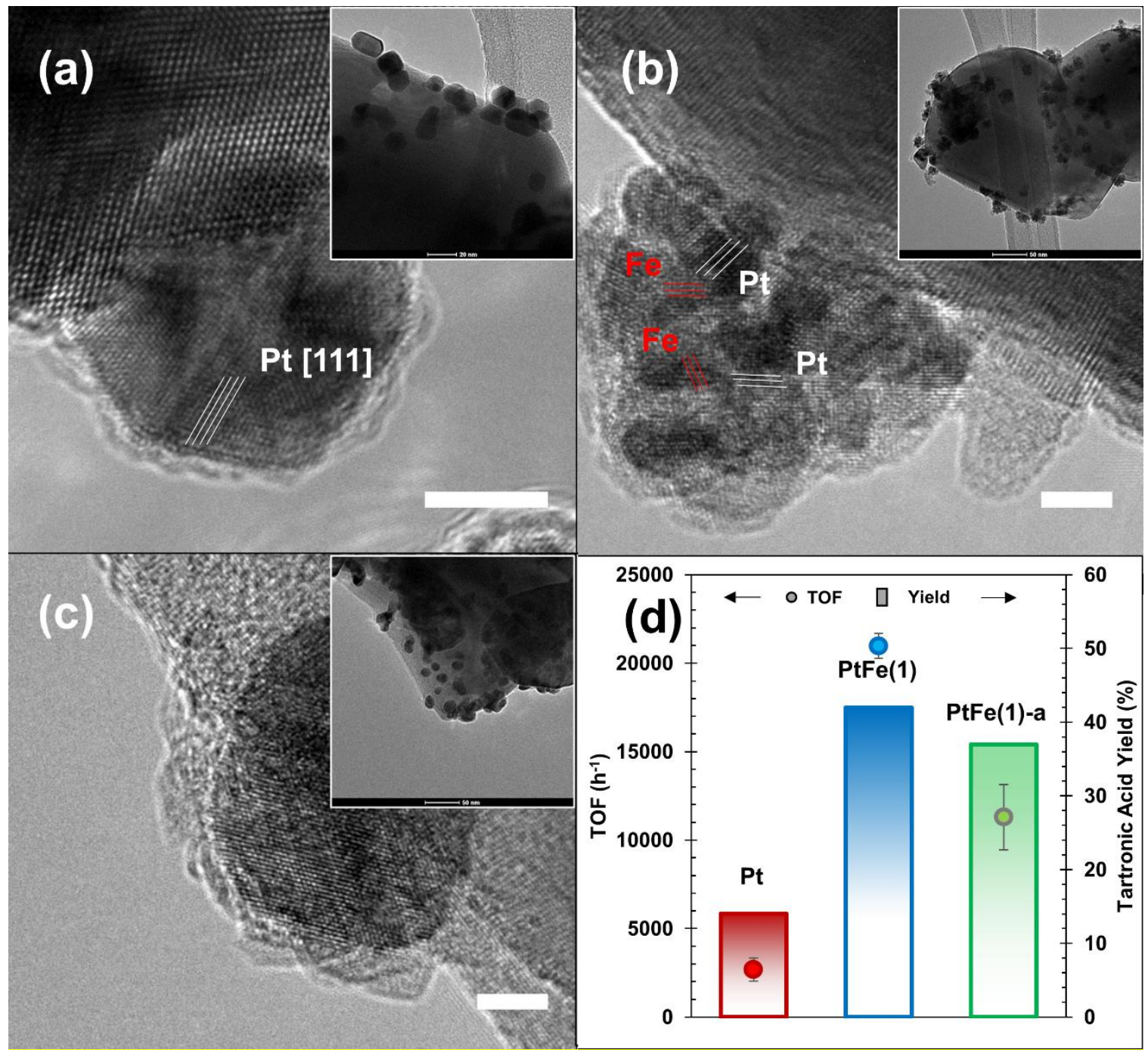

Figure 3. HR-TEM images and glycerol oxidation performances of $\mathrm{Pt}, \mathrm{PtFe}(1)$ and $\mathrm{PtFe}(1)-\mathrm{a}$ catalysts. HR-TEM images of (a) Pt, (b) PtFe(1), (c) PtFe(1)-a with insets at lower magnification. White bars indicate $5 \mathrm{~nm}$. Experimental conditions for (d): TOF values (circles) were calculated based on $0.5-2 \mathrm{~h}$ reaction results at $70{ }^{\circ} \mathrm{C}$ (conversion $<20 \%$ ), tartronic acid yield (bars) was 
obtained at $24 \mathrm{~h}$ reaction time at $70{ }^{\circ} \mathrm{C}$. Glycerol concentration: $0.43 \mathrm{kmol} / \mathrm{m}^{3}, \mathrm{NaOH} / \mathrm{glycerol}$ molar ratio: 4.0, catalyst amount: $2.2 \mathrm{~kg} / \mathrm{m}^{3}, \mathrm{O}_{2}$ pressure: $0.1 \mathrm{MPa}$, refer Experimental Section for other experimental details.

\subsection{Lattice Mismatched Growth Mechanism of Bimetallic PtFe Clusters}

The preliminary results on lattice mismatched bimetallic PtFe catalysts motivated us to carry out further detailed studies to understand the mechanism of lattice mismatch growth, the fundamental structure-activity behavior of such crystals and how the bimetallic structures influence catalytic oxidation reactions. The possible mechanism of cluster formation during solvothermal synthesis was first investigated by doping various amounts of $\mathrm{Fe}$ species to Pt structures ranging from 1/0.5 to $1 / 2$ of $\mathrm{Pt} / \mathrm{Fe}$ molar ratios. This was followed by a detailed rate analysis of glycerol oxidation on selected PtFe and Pt catalysts.

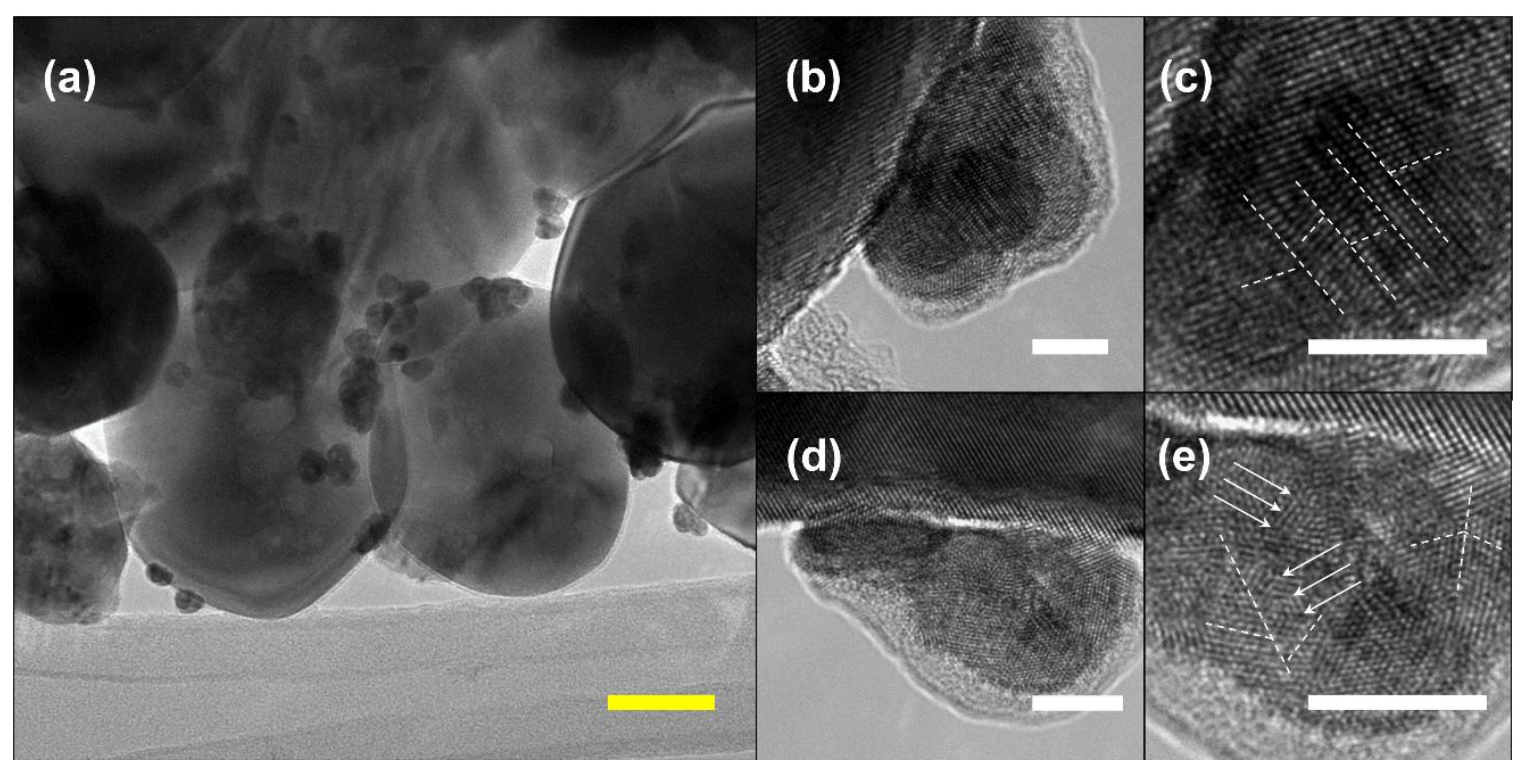

Figure 4. TEM images $\mathrm{PtFe}(0.5)$ catalyst sample. Yellow and white bars indicate $50 \mathrm{~nm}$ and 5 $\mathrm{nm}$ respectively.

HR-TEM images of bimetallic samples containing 1/0.5, 1/1, 1/2 molar Pt/Fe ratios, shown in Figures 4-6, reveal the possible growth mechanism of bimetallic PtFe 
heteroclusters. When the Fe content is small $[\mathrm{PtFe}(0.5)$, Figure 4 (a)], we find that the twin phases of $\mathrm{Pt}$ and $\mathrm{Fe}$ are dominant within the bimetallic sample. To illustrate this aspect, detailed surface atomic arrangement of $\mathrm{PtFe}(0.5)$ sample is shown in Figures 4 (b, c). The twin phases are clearly enclosed by (111) and (100) crystal facets of $f c c$ PtFe structure. Although heterocluster structure is not obvious at this stage, further inspection [see Figure 4 (c)] shows that PtFe surface planes with different orientations start to form. White arrows indicate the possible orientation of boundaries among twin domains. A similar surface geometry is also observed at other regions [see Figures $4(\mathrm{~d}, \mathrm{e})$ ] within the same catalyst sample.

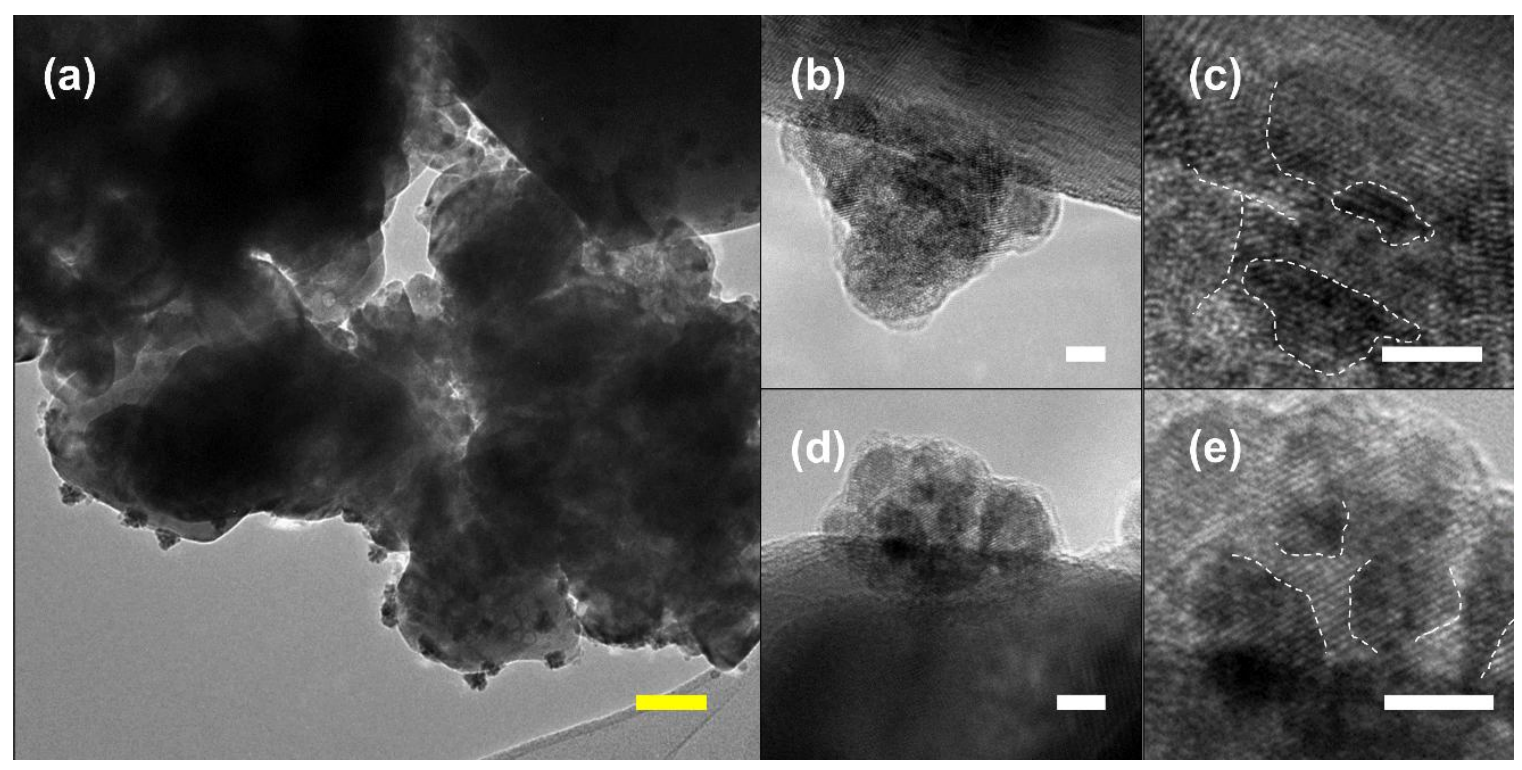

Figure 5. TEM images PtFe(1) catalyst sample. Yellow and white bars indicate $50 \mathrm{~nm}$ and $5 \mathrm{~nm}$ respectively.

At higher Fe content $[\mathrm{PtFe}(1)]$, the heterogeneity of the bimetallic clusters increases, as manifested in the diminution of the Pt (111) and (100) surface planes. As seen in Figure 5 (a), PtFe heterocluster structures are prevalent at several different sample regions. The PtFe(1) sample exhibits a completely disordered cluster-in-cluster geometry rather than 
$f c c$ structures.[47] HR-TEM of two selected regions [Figures 5 (b, d)] further confirm the existence of bimetallic heteroclusters on $\mathrm{CeO}_{2}$ support. Most interestingly, clusters in this sample have clear boundaries [white lines in Figures 5 (c, e)]. This observation implies that when more Fe species exist in bimetallic PtFe clusters, large lattice mismatch might be the dominant factor that governs the growth of nanoparticles.

Two major mechanisms are discussed in the literature for bimetallic crystal growth. One considering etching effects (of $\mathrm{Fe}^{3+}, \mathrm{O}_{2}$, etc) during Pt nanoparticle growth[49] while another proposes anisotropic growth with twin crystals.[50] In our case, we postulate the following mechanisms: (i) at the initial growth stage of bimetallic PtFe clusters, $\mathrm{Fe}^{3+}$ etching is significant. It has been already shown that noble metal cations are reduced faster than non-noble ones in the presence of DMF (a weak reductive agent).[7] When $\mathrm{Pt}^{2+}$ is reduced in the presence of DMF under hydrothermal conditions, the etching effect, also called "galvanic displacement" between existing $\mathrm{Fe}^{3+}$ cations and surface $\mathrm{Pt}^{0}$ results in the formation of $\mathrm{Fe}^{2+}$ and $\mathrm{Pt}^{2+}$.[51] Due to the preferred adsorption of $\mathrm{Fe}^{3+}$ on $\mathrm{Pt}(100)$ surface plane, the "chemical corrosion" of $\mathrm{Pt}^{0}$ to $\mathrm{Pt}^{2+}$ species occurs that in turn affects the surface geometry of Pt nanoparticles.[48] In other words, the regular or organized growth of Pt clusters is disturbed by the presence of Fe species. Thus anisotropic growth and novel structures of bimetallic PtFe can be possibly be obtained. (ii) $\mathrm{Fe}^{2+}$ will also be reduced to $\mathrm{Fe}^{0}$ by DMF, although at a much lower rate compared with $\mathrm{Pt}^{2+}$ reduction. In a parallel pathway, (iii) $\mathrm{Fe}^{0}$ can also be oxidized (displaced) by $\mathrm{Pt}^{2+}$ to $\mathrm{Fe}^{2+}$ in $\mathrm{DMF}$ medium. As a result of (i)-(iii), concave structures can be generated as a template or core material for further growth of PtFe crystals. 


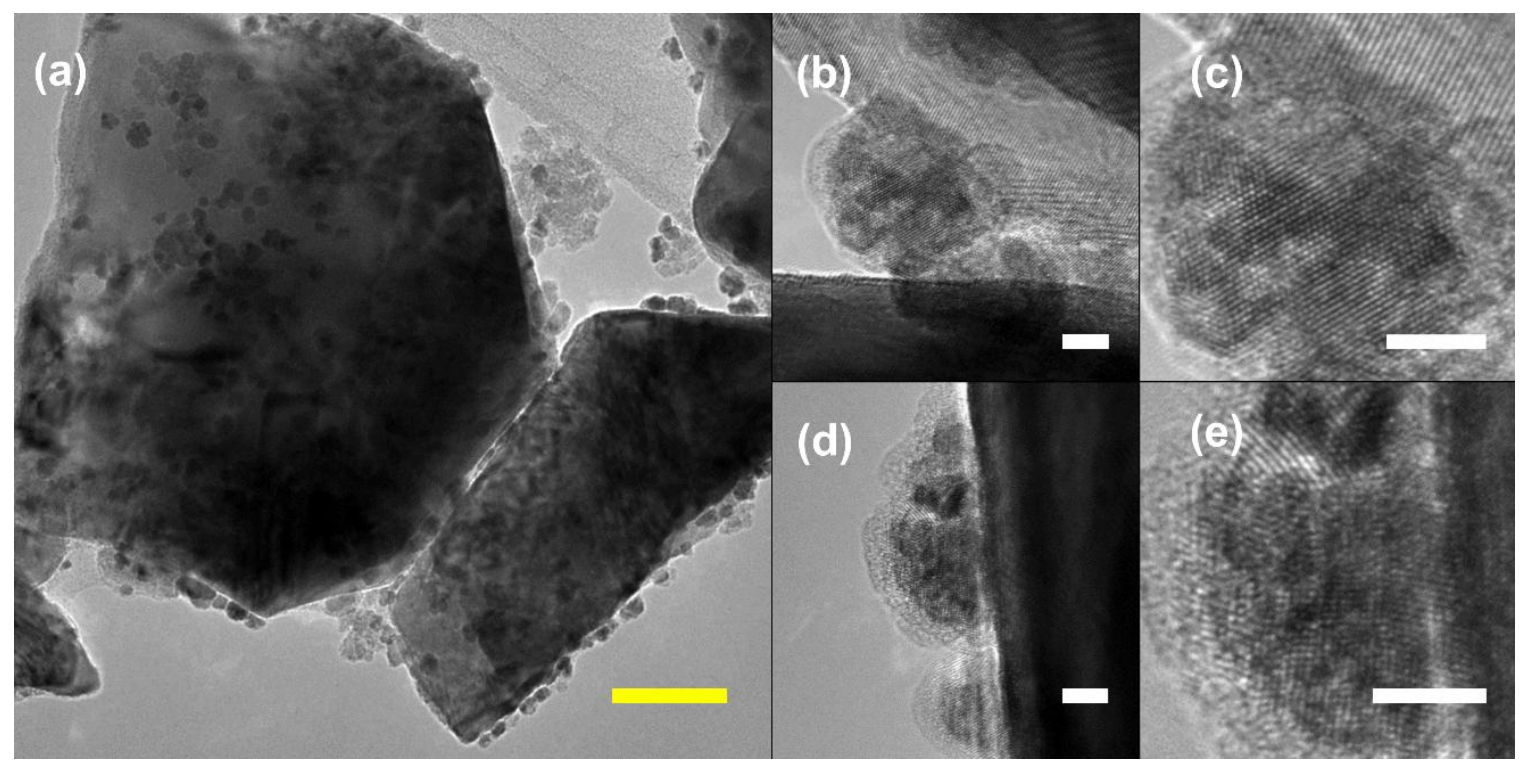

Figure 6. TEM images $\mathrm{PtFe}(2)$ catalyst sample. Yellow and white bars indicate $50 \mathrm{~nm}$ and $5 \mathrm{~nm}$ respectively.
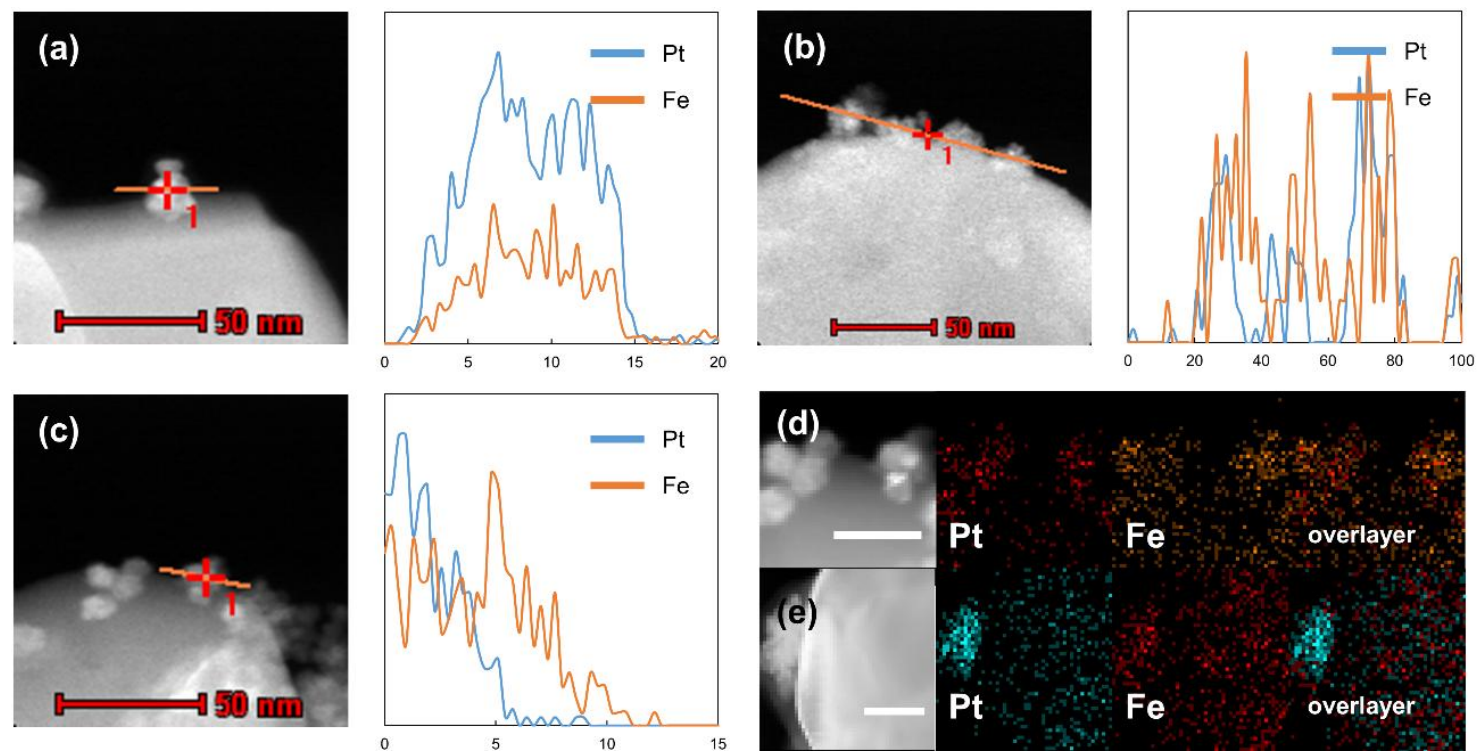

Figure 7. Element line scan and mapping for different $\mathrm{PtFe}$ samples. Line scan of (a) $\mathrm{PtFe}(0.5)$, (b) $\mathrm{PtFe}(1)$ and (c) $\mathrm{PtFe}(2)$ samples; (d) and (e): element mapping of PtFe(1) sample (white bars indicate $20 \mathrm{~nm})$.

The 'etching' mechanism applies only for the formation of well-defined Pt surface planes as $\mathrm{Fe}^{3+}$ was often added as a strong etchant rather than a species participating in bimetallic crystal growth.[52] Hence, such a mechanism is not consistent with the 
formation of bimetallic PtFe heterocluster structures in this work. However, the second mechanism, anisotropic twin crystal growth also known as coalescence mechanism, reported recently for $\mathrm{Pt}, \mathrm{Au}$ and $\mathrm{Pd}$ bimetallic nanocrystals, appears to explain our experimental findings.[53] Detailed inspection of HR-TEM images in Figures 4 (c, e) [PtFe(0.5) sample] and Figures 5 (c, e) [PtFe(1) sample] further confirms the existence of the twined eores structures. In particular, the dash lines show the boundaries of adjacent twin structures, while arrows explain the orientations where crystals tend to grow. These observations suggest that $\mathrm{Fe}^{0}$ species also act as a template for crystal growth because of (iv) $\mathrm{Fe}^{2+}$ reduction to $\mathrm{Fe}^{0}$ in $\mathrm{DMF}$ solution. As a result, anisotropic growth induced by lattice mismatch is dominant on the surface of the twined structures formed initially. Therefore, we propose that lattice strain between template structures and the newly formed shells contribute to the final geometry of the bimetallic PtFe clusters with enhanced heterogeneity and surface index. This alternative mechanistic hypothesis is further supported by the surface geometry presented in Figure 6 [PtFe(2) sample] and additional TEM images of the three samples presented in the supplementary information (Figure S2).

\subsection{Pt and Fe Element Dispersion within Bimetallic Clusters}

The relative rates of reduction of (a) $\mathrm{Pt}^{2+}$ and (b) $\mathrm{Fe}^{3+}$ during crystal formation are also of interest. This is important because the difference in (a) and (b) might lead to distinct surface morphologies of PtFe crystals, such as alloys or core-shells, which could exhibit different surface catalytic activities. Element line scan analysis shown in Figure 7 was carried out for this purpose. When small amounts of Fe is present in Pt clusters $[\mathrm{PtFe}(0.5)$, Figure 7 (a)], bimetallic alloy structures were confirmed by line scan. However, as the Fe 
content increases, more Fe species tend to deposit on Pt surfaces. In other words, although alloy PtFe structures still exist $[\mathrm{PtFe}(2)$, Figure 7 (c)], extra Fe species tend to deposition on the surface alloy structures (see Figure 7). This observation suggests that, when more $\mathrm{Fe}^{3+}$ and $\mathrm{Fe}^{2+}$ species are present during catalyst synthesis, the galvanic displacement is so significant that the overall reduction rate of Fe species is slow and the Fe species tend to deposit on the Pt surface. In fact, element line scan in Figures 7 (a)-(c) also provides such quantitative assessment of both Pt and Fe content in catalyst samples. The element spectra clearly show that the strength of Fe signals with increasing Fe to $\mathrm{Pt}$ ratios from $\mathrm{PtFe}(0.5)$ to $\mathrm{PtFe}(2)$ samples. It is clearly shown that both $\mathrm{Pt}$ (blue) and $\mathrm{Fe}$ (orange) exist inside bimetallic crystals, which suggest alloy structures are presented in all cases. It is important to point out that, Pt and Fe signals fluctuate with different positions within one selected cluster. This information indicates that the surface of clusters is not smooth due to high surface index numbers of bimetallic particles, which supports our hypothesis on heterocluster structures and is in good agreement with TEM images shown in Figures 2-6. To further confirm the heteroclustered structures of PtFe(1) catalyst, additional element mapping is also provided and shown in Figures 7 (d) and (e). Pt and Fe elements are mapped for selected region and presented in different colors. It can be seen that both Pt and Fe element are present but not evenly distributed within selected nanoparticles. Both line scan and element mapping support the hypothesis that PtFe nanoclusters tend to grow in an anisotropic fashion thus high surface indexed clusters can be formed.

\subsection{Kinetic Modeling on Pt and PtFe Catalysts}

Table 1. Glycerol oxidation on $\mathrm{PtFe}(0.5), \mathrm{PtFe}(1)$ and $\mathrm{PtFe}(2)$ catalysts 


\begin{tabular}{lccccc}
\hline \multirow{2}{*}{ Catalyst } & \multirow{2}{*}{$\mathrm{X}(\%)$} & Glyceric & Tartronic & Lactic & Others \\
\hline $\mathrm{PtFe}(0.5)$ & 42.7 & 75.2 & 12.1 & 9.6 & 3.3 \\
$\mathrm{PtFe}(1)$ & 57.1 & 71.1 & 15.8 & 6.3 & 7.1 \\
$\mathrm{PtFe}(2)$ & 54.1 & 77.6 & 12.2 & 3.3 & 5.3 \\
\hline
\end{tabular}

T: $60{ }^{\circ} \mathrm{C}$, reaction time: $2 \mathrm{~h}$, glycerol concentration: $0.43 \mathrm{kmol} / \mathrm{m}^{3}, \mathrm{NaOH} / \mathrm{glycerol}$ molar ratio: 4.0, catalyst amount: $2.2 \mathrm{~kg} / \mathrm{m}^{3}, \mathrm{O}_{2}$ pressure: $0.1 \mathrm{MPa}$, other experimental details refer to Experimental Section.

Next, the structure-dependent kinetics on bimetallic PtFe catalysts were investigated systematically for oxidation of glycerol. It was ensured that the experiments for kinetic modeling were carried out at conditions with negligible mass transfer limitations as indicated by quantitative analysis (see supporting information for detailed calculations). We find that $\mathrm{PtFe}(1)$ catalyst shows slightly better glycerol conversion and tartronic acid selectivity compared to the other two $\mathrm{PtFe}(0.5)$ and $\mathrm{PtFe}(2)$ samples (Table 1). Therefore, further kinetic studies were focused on monometallic Pt and bimetallic PtFe(1) catalyst samples. In particular, concentration-time profiles at $60{ }^{\circ} \mathrm{C}, 70{ }^{\circ} \mathrm{C}$ and $80{ }^{\circ} \mathrm{C}$ on these two catalysts were obtained. As shown in Figure 8, when the temperature is increased from 60 to $80{ }^{\circ} \mathrm{C}$, the initial rate of glycerol consumption is enhanced by almost three folds on $\mathrm{Pt}$ catalyst, while on $\mathrm{PtFe}(1)$ catalyst this value is approximately two fold. Glyceric acid is the dominant product on both catalysts during the $6 \mathrm{~h}$ reaction time. However, the concentration of tartronic acid is about 3-6 times higher on bimetallic $\mathrm{PtFe}(1)$ compared to $\mathrm{Pt}$ catalyst. In addition, we also find that lactic acid concentration is enhanced significantly at higher reaction temperatures. 


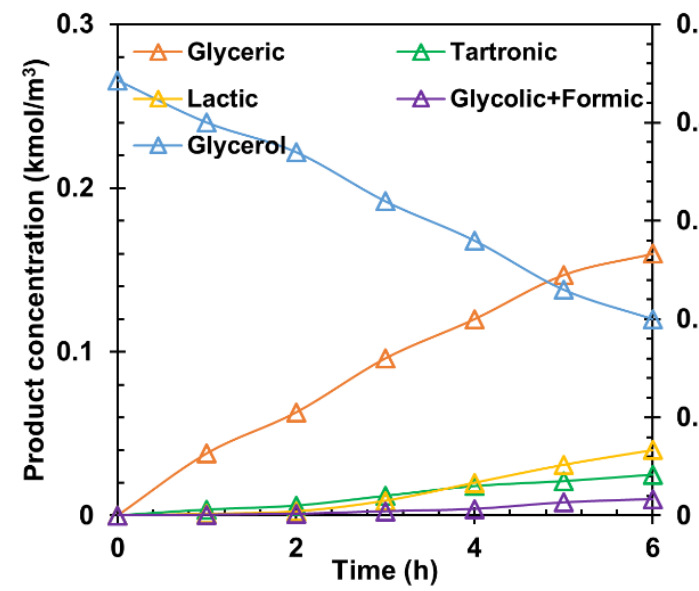

(a)

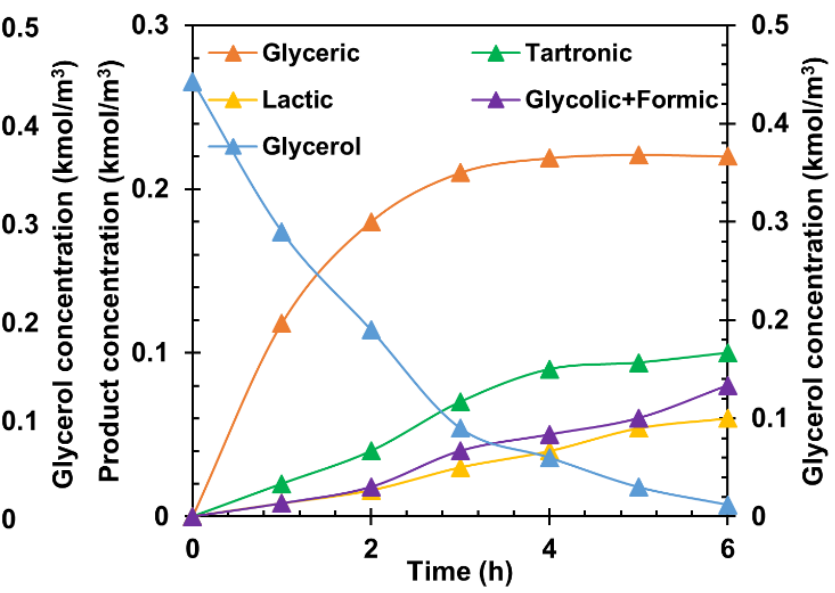

(d)

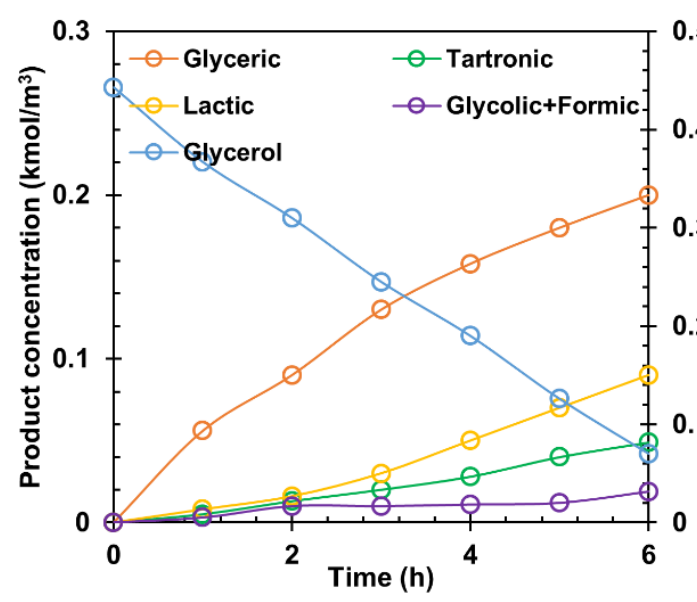

(b)

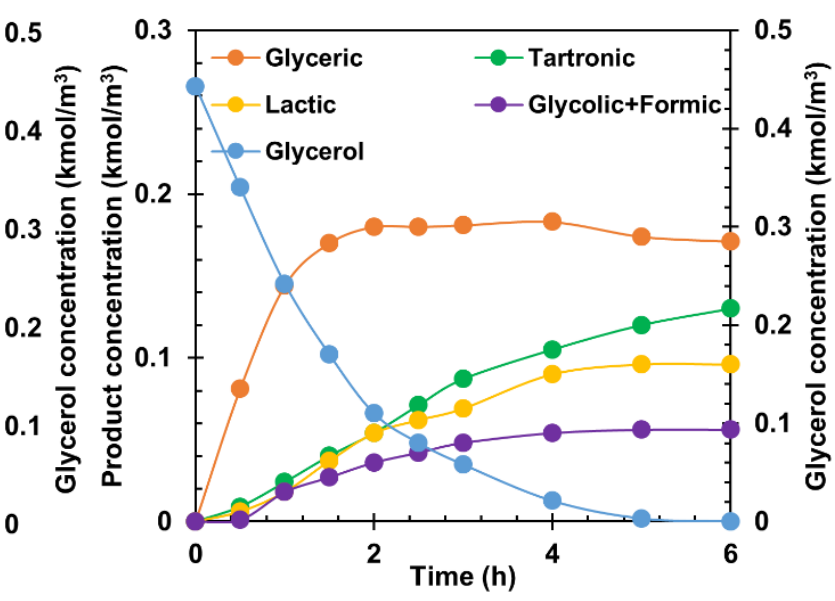

(e)

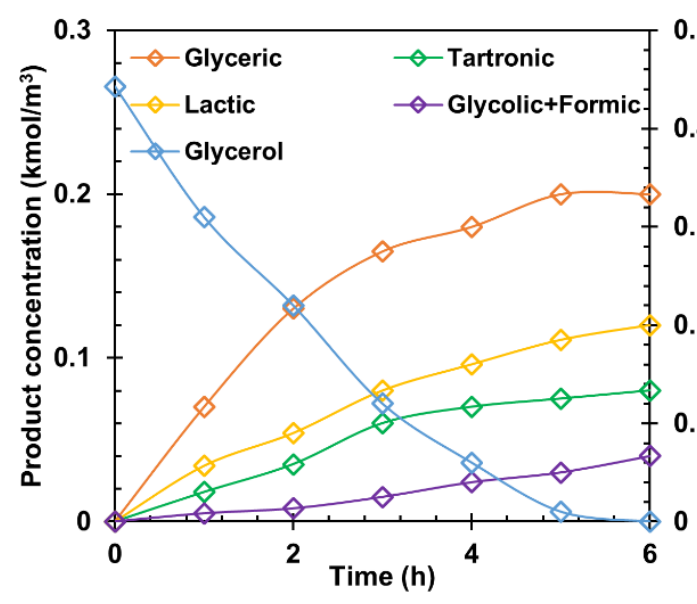

(c)

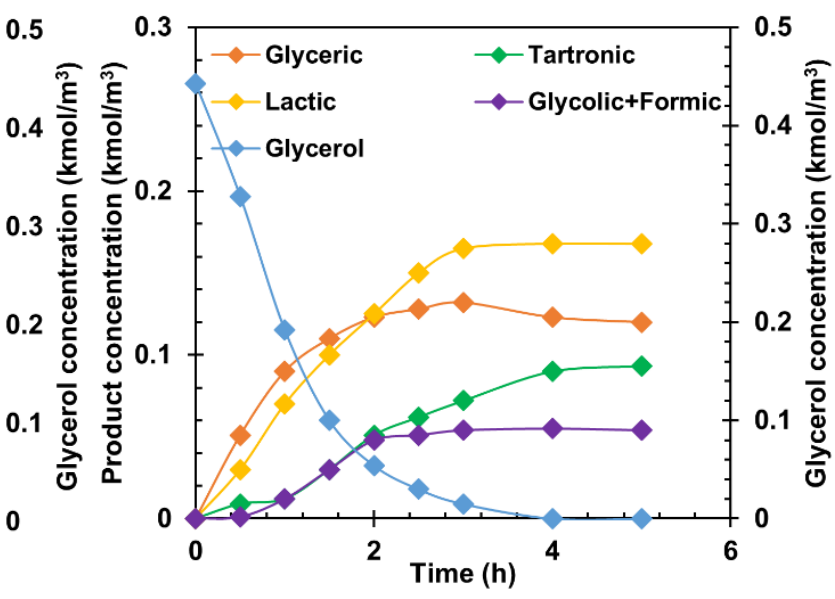

(f)

Figure 8. Concentration-time profiles of glycerol conversion on $\mathrm{Pt} / \mathrm{CeO}_{2}(\mathrm{a}-\mathrm{c}$, hollow) and

$\mathrm{PtFe}(1) / \mathrm{CeO}_{2}$ (d-e, solid). $60{ }^{\circ} \mathrm{C}$ : triangle; $70{ }^{\circ} \mathrm{C}$ : round; $80{ }^{\circ} \mathrm{C}$ : diamond. Experimental conditions: 
glycerol concentration: $0.43 \mathrm{kmol} / \mathrm{m}^{3}, \mathrm{NaOH} /$ glycerol molar ratio: 4.0 , catalyst amount: $2.2 \mathrm{~kg} / \mathrm{m}^{3}$, $\mathrm{O}_{2}$ pressure: 0.1 MPa, refer Experimental Section for other experimental details.

Table 2. Rate constants on Pt and PtFe(1) catalysts

\begin{tabular}{ccccc}
\hline Catalyst & $\begin{array}{c}\text { Rate constant } \\
\left(10^{-2} \mathrm{~m}^{3} / \mathrm{kg}_{\mathrm{cat}} / \mathrm{h}\right)\end{array}$ & $60{ }^{\circ} \mathrm{C}$ & $70{ }^{\circ} \mathrm{C}$ & $80{ }^{\circ} \mathrm{C}$ \\
\hline \multirow{2}{*}{$\mathrm{Pt}$} & $k_{1}$ & 0.48 & 0.78 & 1.28 \\
& $k_{2}$ & 0.29 & 0.49 & 0.64 \\
& $k_{3}$ & 0.08 & 0.24 & 0.54 \\
& $k_{4}$ & 0.02 & 0.04 & 0.14 \\
$\mathrm{PtFe}(1)$ & $k_{1}$ & 1.81 & 2.48 & 2.85 \\
& $k_{2}$ & 0.46 & 0.69 & 0.84 \\
& $k_{3}$ & 0.28 & 0.74 & 1.89 \\
& $k_{4}$ & 0.46 & 0.59 & 0.79 \\
\hline
\end{tabular}

Catalyst amount: $2.2 \mathrm{~kg} / \mathrm{m}^{3}$

With these experimental observations, systematic kinetic modeling was carried out. The purpose of kinetic analysis is to obtain quantitative assessment of the role of $\mathrm{Fe}$ in $\mathrm{Pt}$ metals in glycerol oxidation. Specific reactions involved in glycerol conversion including (1) oxidation of glycerol (A) to glyceric acid (B, first step oxidation, $\left.r_{1}\right)$, (2) oxidation of glyceric to tartronic acid (C, second step oxidation, $\left.r_{2}\right)$, (3) oxidation of glycerol to lactic acid (D, $\left.r_{3}\right)$ and (4) glycerol to formic and glycolic acids (E, C-C cleavage and oxidation, $r_{4}$ ) were considered for this part of study (see A to E in Figure 9 inset). The lumped reaction pathways have already been well described in Figure 1. Moreover, rate constants for $r_{1}-r_{4}$ (Table 2) were regressed by fitting the observed concentration-time data (see fittings in Figures 10 and S3). We used the kinetic analysis for calculation of activation barriers. As shown in Figure 9, the activation barriers for $r_{l}$ (glycerol to glyceric acid) and $r_{2}$ (glyceric to tartronic acid) on monometallic Pt catalysts are $48.4 \mathrm{~kJ} / \mathrm{mol}$ and 38.3 
$\mathrm{kJ} / \mathrm{mol}$, respectively, the value ranges of which are well consistent with previous reports on the same reaction. $[28,54,55]$ Importantly, we find that the presence of Fe lowers the activation energies for primary and secondary oxidation on Pt catalyst. The activation barrier for lactic acid formation is almost unchanged but that for $\mathrm{C}-\mathrm{C}$ cleavage is much lower on bimetallic $\mathrm{PtFe}(1)$ catalyst. In addition, we also find that the activation energy for C-C cleavage $\left(r_{4}\right)$ is also significantly reduced on bimetallic $\mathrm{PtFe}(1)$ catalyst. This result suggests that, due to lower oxidation barrier when $\mathrm{Fe}$ is present, formation of $-\mathrm{C}=\mathrm{O}$ group is much more easier on $\mathrm{PtFe}(1)$ catalysts. C-C cleavage via retro-aldolization therefore only needs to overcome lower activation barrier compared with Pt catalysts, which might lead to more C-C cleavage products (glycolic and formic acid). However, the overall reaction rate for $\mathrm{C}-\mathrm{C}$ cleavage $\left(r_{4}\right)$ is much lower than primary and secondary oxidation $\left(r_{1}, r_{2}\right)$ thus tartronic acid is still the dominant oxidation product on $\mathrm{PtFe}(1)$ catalyst. Therefore, it is clear that bimetallic PtFe catalysts enhance the tartronic acid formation by significantly lowering the activation barriers for primary and secondary oxidations. We also compared the proposed PtFe(1) catalyst with leading catalyst systems in literature (see Table S2). It is seen from comparison that $\mathrm{PtFe}(1)$ catalyst outperforms all existing systems in terms of TOF values and show very good selectivity towards tartronic acid. 


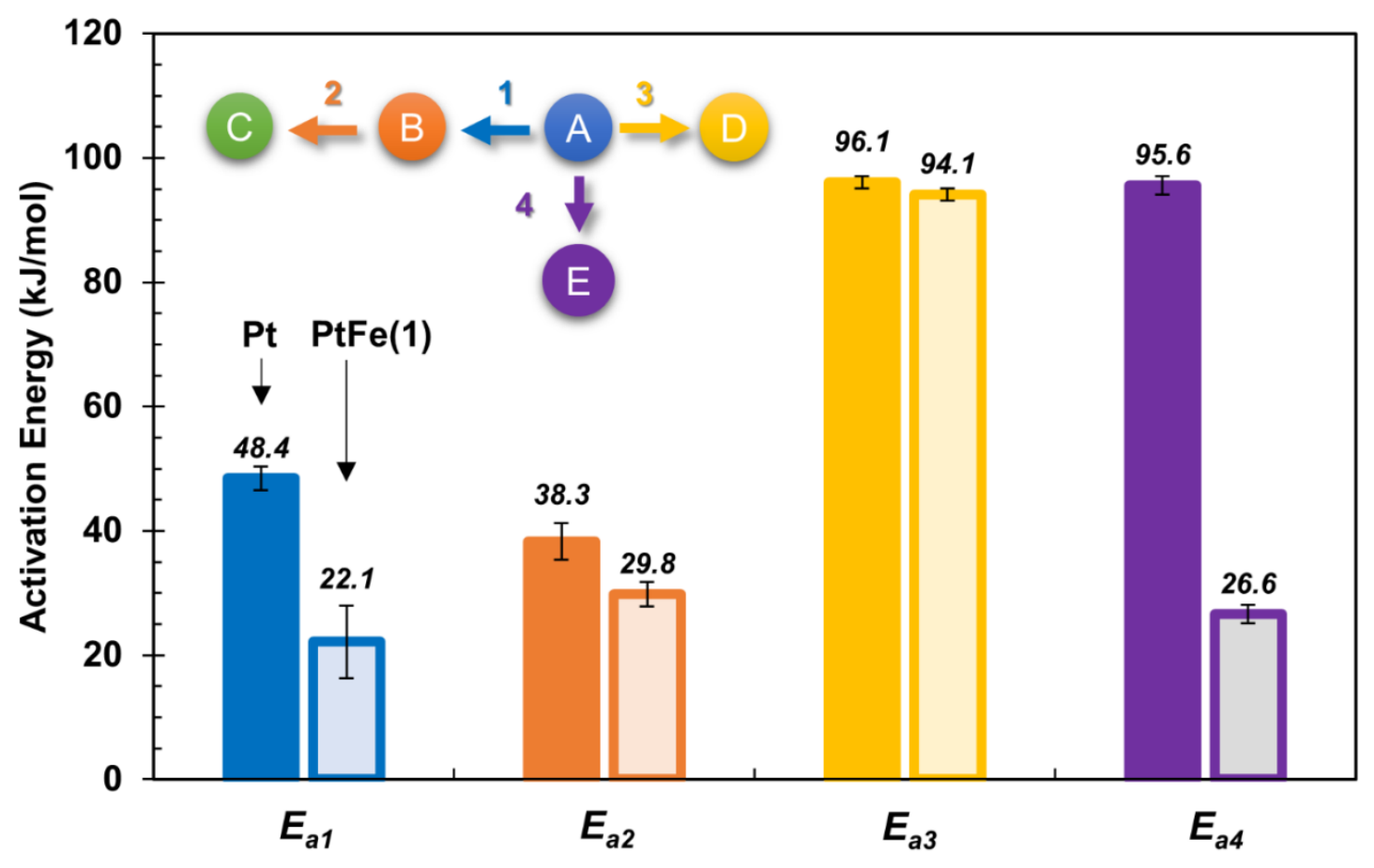

Figure 9. Activation barriers for glycerol conversion in presence of $\mathrm{Pt}$ (solid bars) and $\mathrm{PtFe}(1)$ (hollow bars) catalysts. [A: glycerol, B: glyceric acid, C: tartronic acid, D: lactic acid, E: formic and glycolic acids; $E_{a l}-E_{a 4}$ represent activation energies for $r_{l}$ (blue), $r_{2}$ (orange), $r_{3}$ (yellow) and $r_{4}$ (purple), respectively]

Based on the experimental evidence and kinetic modeling presented in this work, we proposed a possible reaction mechanism for glycerol oxidation on bimetallic PtFe and monometallic Pt catalysts (Figure 11). It is known that strong adsorption of $-\mathrm{C}=\mathrm{O}$ group on noble metal surfaces (e.g. Pt, Pd) often prevents further (oxidation) reactions.[11, 12, 28] The high tendency for decarboxylation or oxidative decarbonylation, due to strong interactions noted in the literature, is known to lead to significant side reactions such as $\mathrm{CO}$ formation and resultant catalyst poisoning at low temperature.[29] Clearly, the strong interaction demands higher activation energy for transformation of $-\mathrm{C}=\mathrm{O}$ groups (in glyceraldehyde or glyceric acid molecules) on monometallic Pt catalyst surface (orange 
route in Figure 11). By doping Fe (promoters) to Pt systems, the binding energy between $-\mathrm{C}=\mathrm{O}$ functional groups and metal atoms is lowered.[56] This favorably decreases the surface coverage of $-\mathrm{C}=\mathrm{O}$ groups, or increases the possibility of catalytic turnovers on metal sites, thus favoring oxidation rates on the catalyst surface (green route in Figure 11). The lower activation barrier for the primary reaction step on bimetallic $\mathrm{PtFe}(1)$ catalyst clearly confirms this hypothesis. The presence of $\mathrm{Fe}$ promotes desorption of $-\mathrm{C}=\mathrm{O}$ groups from active sites thus enhancing secondary oxidation reactions. Therefore, tartronic acid selectivity is much higher on bimetallic PtFe(1) nanocatalyst. The stability of the PtFe catalyst was also investigated by catalyst recycle studies at $70{ }^{\circ} \mathrm{C}$. We find that the conversion of glycerol on PtFe(1) catalyst displays a slight decrease from $100 \%$ to $95 \%$, with selectivity towards tartronic acid decreasing from $32 \%$ to $22 \%$, after the third recycle (Figure 12). TEM images of spent PtFe(1) catalyst are shown in Figure 13, where it is found that the structural properties remain almost unchanged after three recycles. In order to understand the plausible reason for catalyst deactivation, XPS and metal leaching tests were further conducted. XPS characterization results show that Pt species in both fresh and used PtFe(1) catalyst samples remain metallic state, while the binding energy for Pt display slightly shift from $71.02 \mathrm{eV}$ to $70.96 \mathrm{eV}$ (see Table S3 for details). Metal leaching tests were also carried for PtFe(1) catalyst by ICP analysis. We observed that the total leaching of Pt and Fe content after three recycles are 3.2\% and $35.7 \%$ respectively. Therefore it is plausible that the deactivation observed with $\mathrm{PtFe}(1)$ catalysts might be due to Fe leaching. 


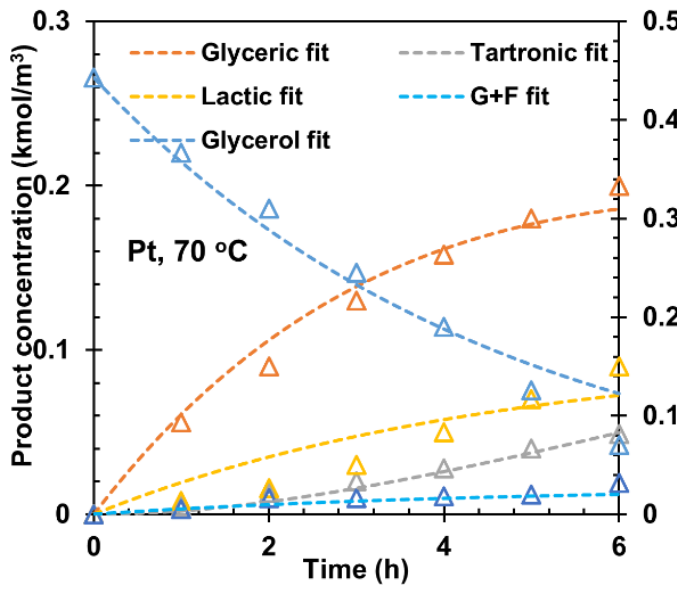

(a)

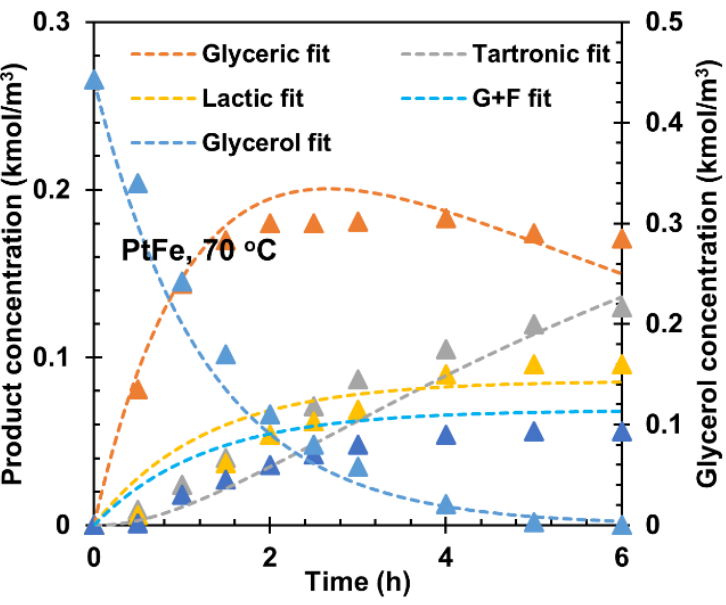

(b)

Figure 10. Fitting with experimental data at $70{ }^{\circ} \mathrm{C}$. (a) $\mathrm{Pt}$, (b) $\mathrm{PtFe}(1)$ catalysts (points:

experimental data; lines: model fitting. Refer to Figure S3 for fitting at other temperatures)

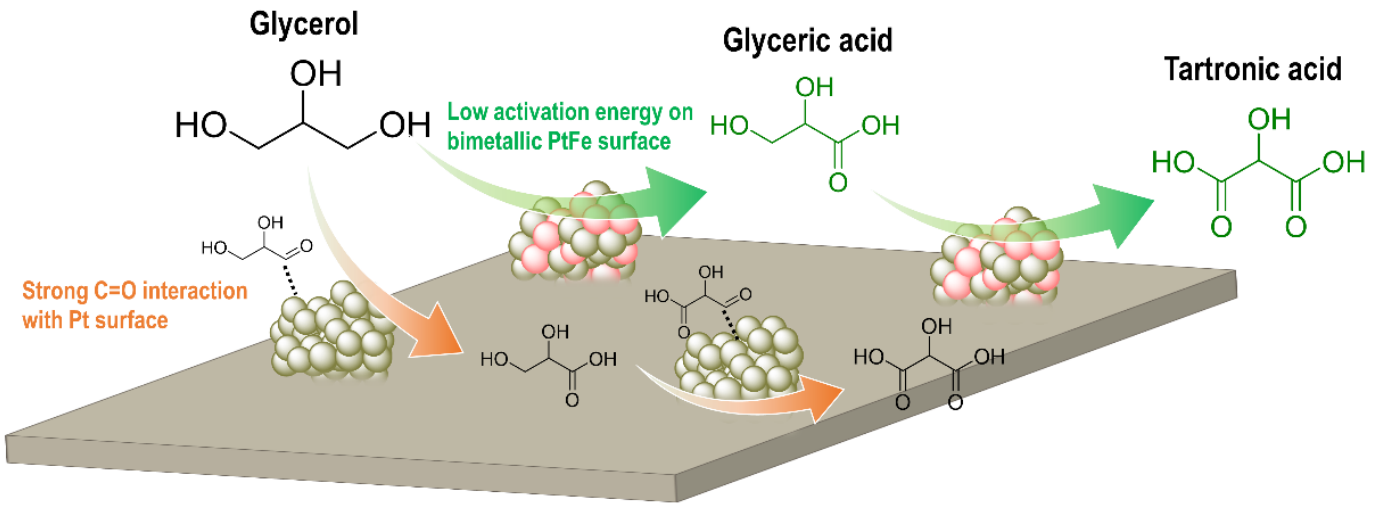

Figure 11. Mechanistic description of glycerol oxidation on PtFe and Pt catalysts 


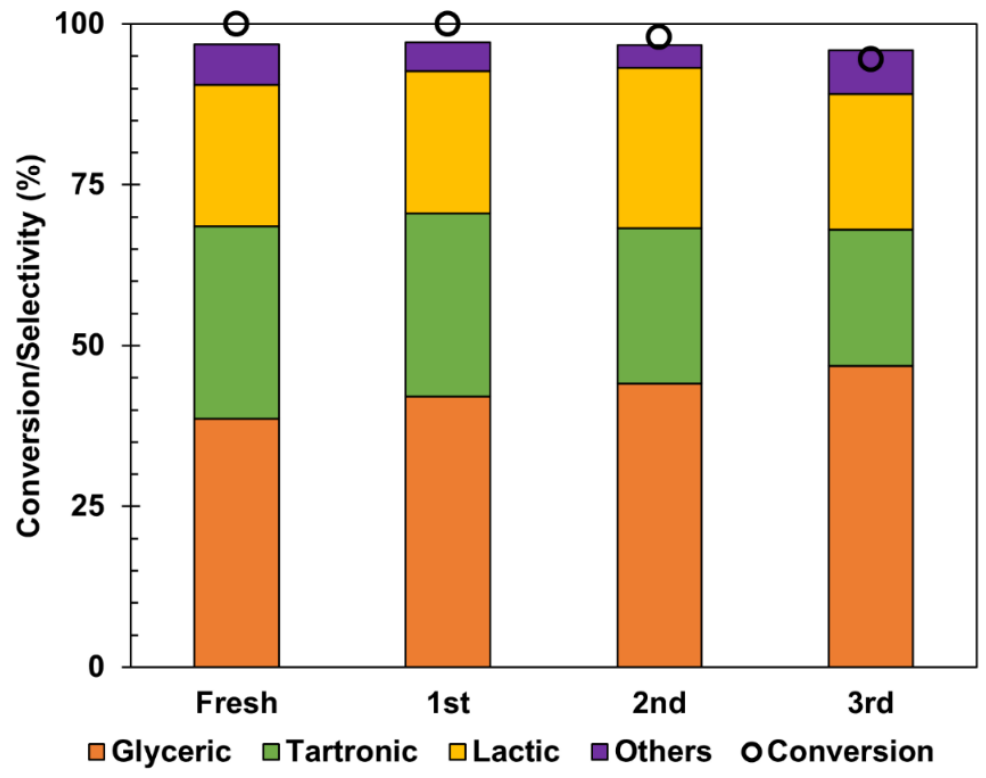

Figure 12. Recycle studies of $\mathrm{PtFe}(1)$ catalyst at $70{ }^{\circ} \mathrm{C}$ (reaction time: $6 \mathrm{~h}$ ).

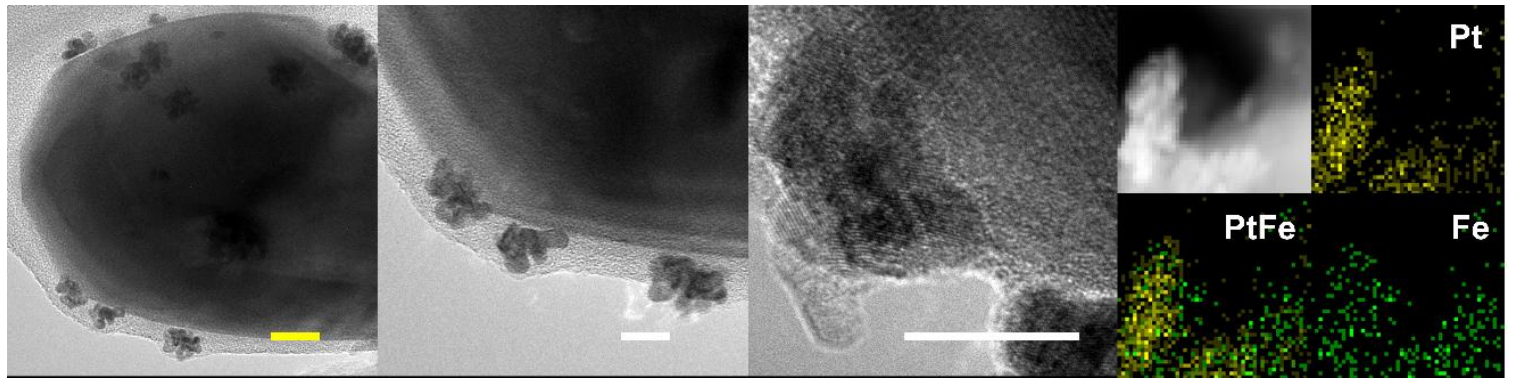

Figure 13. TEM images and element mapping of spent $\mathrm{PtFe}(1)$ catalyst after three recycles (Yellow and white bars indicate $20 \mathrm{~nm}$ and $10 \mathrm{~nm}$ respectively).

Table 3. Conversion and selectivity for sorbitol oxidation on $\mathrm{Pt}$ and $\mathrm{PtFe}(1)$ catalysts

\begin{tabular}{llllllll}
\hline \multirow{2}{*}{ Catalyst } & $\mathrm{X}(\%)$ & \multicolumn{7}{c}{ Selectivity (\%) } \\
\cline { 3 - 8 } & & glyceric & tartronic & gluconic & glucaric & oxalic & formic \\
\hline $\mathrm{Pt} / \mathrm{CeO}_{2}$ & $32.4 \%$ & 48.9 & 13.1 & 5.4 & 5.2 & 11.0 & 5.9 \\
$\mathrm{PtFe}(1) / \mathrm{CeO}_{2}$ & $100 \%$ & 36.3 & 11.9 & 11.4 & 18.3 & 9.0 & 11.0 \\
\hline
\end{tabular}

Experimental conditions: $0.05 \mathrm{~g}$ of solid catalyst, $25 \mathrm{~mL}$ aqueous solution containing $0.5 \mathrm{~g}$ sorbitol and $1.7 \mathrm{~g} \mathrm{NaOH}, 0.1 \mathrm{MPa}_{2}, 70{ }^{\circ} \mathrm{C}, 6 \mathrm{~h}$ 
In addition, we also tested the $\mathrm{PtFe}(1)$ catalyst for the oxidation of sorbitol, a $\mathrm{C}_{6}$ polyol, at $70{ }^{\circ} \mathrm{C}$. Complete conversion was observed after $6 \mathrm{~h}$ reaction time and the major products included glyceric $(36 \%)$, tartronic $(12 \%)$, gluconic $(11 \%)$, glucaric $(18 \%)$, oxalic (9\%) and formic (11\%) acids. It is clear that PtFe(1) outperforms Pt catalyst for sorbitol oxidation in terms of conversion and selectivity. This result suggests that the proposed bimetallic $\mathrm{PtFe}(1)$ can also effectively catalyze oxidation of other biomass polyols under mild conditions.

\section{Conclusion}

By using the large lattice mismatch between Pt and Fe metals, anisotropic growth of bimetallic PtFe nanoclusters can be achieved. The resultant bimetallic clusters display high index numbers and exhibits synergistic oxidation activity and selectivity for liquid phase glycerol oxidation. Kinetic modeling is used as a tool to reveal that the presence of Fe species in Pt catalysts decrease the activation barriers for both primary and secondary oxidation reactions. These results show that lattice mismatched growth results in high index bimetallic PtFe nanocatalysts, which can efficiently produce valuable hydroxyl dicarboxylic acids from various biomass feedstocks indicating clear potential of wider application of bimetallic catalysts for catalytic oxidation reactions.

\section{Acknowledgement}

This work is supported by NSF/EPA grant CHE-1339661.

\section{References}

[1] X.M. Lu, M. Rycenga, S.E. Skrabalak, B. Wiley, Y.N. Xia, Chemical Synthesis of Novel Plasmonic Nanoparticles, Annu Rev Phys Chem, 60 (2009) 167-192. 
[2] N.S. Porter, H. Wu, Z.W. Quan, J.Y. Fang, Shape-Control and Electrocatalytic Activity-

Enhancement of Pt-Based Bimetallic Nanocrystals, Accounts Chem Res, 46 (2013) 1867-1877.

[3] P. Gallezot, Conversion of biomass to selected chemical products, Chem Soc Rev, 41 (2012) 1538-1558.

[4] J.C. Serrano-Ruiz, J.A. Dumesic, Catalytic routes for the conversion of biomass into liquid hydrocarbon transportation fuels, Energ Environ Sci, 4 (2011) 83-99.

[5] J.B. Wu, P.P. Li, Y.T. Pan, S. Warren, X. Yin, H. Yang, Surface lattice-engineered bimetallic nanoparticles and their catalytic properties, Chem Soc Rev, 41 (2012) 8066-8074.

[6] R.G. Chaudhuri, S. Paria, Core/Shell Nanoparticles: Classes, Properties, Synthesis

Mechanisms, Characterization, and Applications, Chem Rev, 112 (2012) 2373-2433.

[7] J.P. Lai, W.X. Niu, R. Luque, G.B. Xu, Solvothermal synthesis of metal nanocrystals and their applications, Nano Today, 10 (2015) 240-267.

[8] Z.N. Wang, Z.Z. Chen, H. Zhang, Z.R. Zhang, H.J. Wu, M.S. Jin, C. Wu, D.R. Yang, Y.D.

Yin, Lattice-Mismatch-Induced Twinning for Seeded Growth of Anisotropic Nano structures, Acs Nano, 9 (2015) 3307-3313.

[9] R.R. Davda, J.W. Shabaker, G.W. Huber, R.D. Cortright, J.A. Dumesic, A review of catalytic issues and process conditions for renewable hydrogen and alkanes by aqueous-phase reforming of oxygenated hydrocarbons over supported metal catalysts, Appl Catal B-Environ, 56 (2005) 171186.

[10] Y.H. Shen, S.H. Zhang, H.J. Li, Y. Ren, H.C. Liu, Efficient Synthesis of Lactic Acid by Aerobic Oxidation of Glycerol on Au-Pt/TiO2 Catalysts, Chem-Eur J, 16 (2010) 7368-7371. [11] E. Sproge, S. Chornaja, K. Dubencovs, S. Zhizhkun, V. Kampars, V. Serga, L. Kulikova, E. Palcevskis, Selective liquid phase oxidation of glycerol to glyceric acid over novel supported $\mathrm{Pt}$ catalysts, J Serb Chem Soc, 78 (2013) 1359-1372.

[12] A.A. Rodriguez, C.T. Williams, J.R. Monnier, Selective liquid-phase oxidation of glycerol over $\mathrm{Au}-\mathrm{Pd} / \mathrm{C}$ bimetallic catalysts prepared by electroless deposition, Appl Catal a-Gen, 475 (2014) 161-168.

[13] X. Jin, L.N. Dang, J. Lohrman, B. Subramaniam, S.Q. Ren, R.V. Chaudhari, LatticeMatched Bimetallic CuPd-Graphene Nanocatalysts for Facile Conversion of Biomass-Derived Polyols to Chemicals, Acs Nano, 7 (2013) 1309-1316.

[14] X. Jin, M. Zhao, J. Shen, W.J. Yan, L.M. He, P.S. Thapa, S.Q. Ren, B. Subramaniam, R.V. Chaudhari, Exceptional performance of bimetallic Pt1Cu3/TiO2 nanocatalysts for oxidation of gluconic acid and glucose with O2 to glucaric acid, J Catal, 330 (2015) 323-329.

[15] W.J. Yan, A. Ramanathan, M. Ghanta, B. Subramaniam, Towards highly selective ethylene epoxidation catalysts using hydrogen peroxide and tungsten-or niobium-incorporated mesoporous silicate (KIT-6), Catal Sci Technol, 4 (2014) 4433-4439.

[16] Y. Onal, S. Schimpf, P. Claus, Structure sensitivity and kinetics of D-glucose oxidation to Dgluconic acid over carbon-supported gold catalysts, J Catal, 223 (2004) 122-133.

[17] A. Abbadi, H. Vanbekkum, Effect of Ph in the Pt-Catalyzed Oxidation of D-Glucose to DGluconic Acid, J Mol Catal a-Chem, 97 (1995) 111-118.

[18] X. Jin, D. Roy, P.S. Thapa, B. Subramaniam, R.V. Chaudhari, Atom Economical AqueousPhase Conversion (APC) of Biopolyols to Lactic Acid, Glycols, and Linear Alcohols Using Supported Metal Catalysts, Acs Sustain Chem Eng, 1 (2013) 1453-1462.

[19] G.L. Brett, Q. He, C. Hammond, P.J. Miedziak, N. Dimitratos, M. Sankar, A.A. Herzing, M. Conte, J.A. Lopez-Sanchez, C.J. Kiely, D.W. Knight, S.H. Taylor, G.J. Hutchings, Selective Oxidation of Glycerol by Highly Active Bimetallic Catalysts at Ambient Temperature under Base-Free Conditions, Angew Chem Int Edit, 50 (2011) 10136-10139.

[20] Z. Zhao, J. Arentz, J. Clomburg, R. Gonzalez, L.A. Pretzer, N. Schweitzer, J.T. Miller, M.S. Wong, Modulating palladium-on-gold catalysis for glycerol oxidation with Pd surface coverage, Abstr Pap Am Chem S, 243 (2012). 
[21] B. Katryniok, H. Kimura, E. Skrzynska, J.S. Girardon, P. Fongarland, M. Capron, R. Ducoulombier, N. Mimura, S. Paul, F. Dumeignil, Selective catalytic oxidation of glycerol: perspectives for high value chemicals, Green Chem, 13 (2011) 1960-1979.

[22] S. Hirasawa, Y. Nakagawa, K. Tomishige, Selective oxidation of glycerol to dihydroxyacetone over a Pd-Ag catalyst, Catal Sci Technol, 2 (2012) 1150-1152.

[23] A. Zalineeva, A. Serov, M. Padilla, U. Martinez, K. Artyushkova, S. Baranton, C. Coutanceau, P. Atanassov, Nano-structured Pd-Sn catalysts for alcohol electro-oxidation in alkaline medium, Electrochem Commun, 57 (2015) 48-51.

[24] A. Villa, N. Dimitratos, C.E. Chan-Thaw, C. Hammond, L. Prati, G.J. Hutchings, Glycerol Oxidation Using Gold-Containing Catalysts, Accounts Chem Res, 48 (2015) 1403-1412.

[25] J.Y. Cai, H. Ma, J.J. Zhang, Z.T. Du, Y.Z. Huang, J. Gao, J. Xu, Catalytic oxidation of glycerol to tartronic acid over Au/HY catalyst under mild conditions, Chinese J Catal, 35 (2014) 1653-1660.

[26] H. Kimura, A. Kimura, I. Kokubo, T. Wakisaka, Y. Mitsuda, Palladium Based Multicomponent Catalytic-Systems for the Alcohol to Carboxylate Oxidation Reaction, Appl Catal a-Gen, 95 (1993) 143-169.

[27] H. Kimura, Oxidation assisted new reaction of glycerol, Polym Advan Technol, 12 (2001) 697-710.

[28] W.B. Hu, B. Lowry, A. Varma, Kinetic study of glycerol oxidation network over Pt-Bi/C catalyst, Appl Catal B-Environ, 106 (2011) 123-132.

[29] C.L. Bianchi, P. Canton, N. Dimitratos, F. Porta, L. Prati, Selective oxidation of glycerol with oxygen using mono and bimetallic catalysts based on $\mathrm{Au}, \mathrm{Pd}$ and Pt metals, Catal Today, 102 (2005) 203-212.

[30] J.M.H. Dirkx, H.S. Vanderbaan, The Oxidation of Gluconic Acid with Platinum on Carbon as Catalyst, J Catal, 67 (1981) 14-20.

[31] J. Suntivich, Z.C. Xu, C.E. Carlton, J. Kim, B.H. Han, S.W. Lee, N. Bonnet, N. Marzari, L.F. Allard, H.A. Gasteiger, K. Hamad-Schifferli, Y. Shao-Horn, Surface Composition Tuning of AuPt Bimetallic Nanoparticles for Enhanced Carbon Monoxide and Methanol Electro-oxidation, J Am Chem Soc, 135 (2013) 7985-7991.

[32] Z.W. Quan, Y.X. Wang, J.Y. Fang, High-Index Faceted Noble Metal Nanocrystals, Accounts Chem Res, 46 (2013) 191-202.

[33] J. Gu, Y.W. Zhang, F. Tao, Shape control of bimetallic nanocatalysts through well-designed colloidal chemistry approaches, Chem Soc Rev, 41 (2012) 8050-8065.

[34] H.W. Wang, C.L. Wang, H. Yan, H. Yi, J.L. Lu, Precisely-controlled synthesis of Au@Pd core-shell bimetallic catalyst via atomic layer deposition for selective oxidation of benzyl alcohol, J Catal, 324 (2015) 59-68.

[35] D. Van Thuan, N.T. Khoa, S.W. Kim, E.J. Kim, S.H. Hahn, Morphology-dependent selective hydrogenation catalysis of hollow AuCu bimetallic nanostructures, J Catal, 329 (2015) 144-150.

[36] A. Tanksale, J.N. Beltramini, J.A. Dumesic, G.Q. Lu, Effect of Pt and Pd promoter on Ni supported catalysts - A TPR/TPO/TPD and microcalorimetry study, J Catal, 258 (2008) 366-377.

[37] C.B. Roy, D.K. Nandi, K.V. Rao, Electrocatalytic Oxidation of Methanol, Ethanol, Isopropanol, Glycol and Glycerol on Pt-Au Electrodes, Indian J Chem A, 24 (1985) 742-744. [38] Z. Zhao, J. Arentz, L.A. Pretzer, P. Limpornpipat, J.M. Clomburg, R. Gonzalez, N.M. Schweitzer, T.P. Wu, J.T. Miller, M.S. Wong, Volcano-shape glycerol oxidation activity of palladium-decorated gold nanoparticles, Chem Sci, 5 (2014) 3715-3728.

[39] L. Wang, W. Zhang, S.J. Zeng, D.S. Su, X.J. Meng, F.S. Xiao, Mg-Al Mixed Oxides Supported Bimetallic Au-Pd Nanoparticles with Superior Catalytic Properties in Aerobic Oxidation of Benzyl Alcohol and Glycerol, Chinese J Chem, 30 (2012) 2189-2197. 
[40] D. Liang, S.Y. Cui, J. Gao, J.H. Wang, P. Chen, Z.Y. Hou, Glycerol Oxidation with Oxygen over Bimetallic Pt-Bi Catalysts under Atmospheric Pressure, Chinese J Catal, 32 (2011) 18311837.

[41] A. Villa, C. Campione, L. Prati, Bimetallic gold/palladium catalysts for the selective liquid phase oxidation of glycerol, Catal Lett, 115 (2007) 133-136.

[42] N. Dimitratos, J.A. Lopez-Sanchez, D. Lennon, F. Porta, L. Prati, A. Villa, Effect of particle size on monometallic and bimetallic $(\mathrm{Au}, \mathrm{Pd}) / \mathrm{C}$ on the liquid phase oxidation of glycerol, Catal Lett, 108 (2006) 147-153.

[43] D. Wang, A. Villa, F. Porta, D.S. Su, L. Prati, Single-phase bimetallic system for the selective oxidation of glycerol to glycerate, Chem Commun, (2006) 1956-1958.

[44] K. Hermann, Crystallography and Surface Structure: An Introduction for Surface Scientists and Nanoscientists, Wiley-VCH Verlag GmbH \& Co. KGaA, Weinheim, Germany, 2011.

[45] X. Jin, B. Subramaniam, R.V. Chaudhari, Activity and Selectivity of Base Promoted Mono and Bimetallic Catalysts for Hydrogenolysis of Xylitol and Sorbitol, in: J.J. Bravo-Suárez, M.

Kidder, K., S. V. (Eds.) Novel Materials for Catalysis and Fuels Processing, American Chemical Society, 2013.

[46] S.T. Oyama, X.M. Zhang, J.Q. Lu, Y.F. Gu, T. Fujitani, Epoxidation of propylene with H-2 and O-2 in the explosive regime in a packed-bed catalytic membrane reactor, J Catal, 257 (2008) $1-4$.

[47] Z.R. Dai, S.H. Sun, Z.L. Wang, Phase transformation, coalescence, and twinning of monodisperse FePt nanocrystals, Nano Lett, 1 (2001) 443-447.

[48] K.E. Elkins, T.S. Vedantam, J.P. Liu, H. Zeng, S.H. Sun, Y. Ding, Z.L. Wang, Ultrafine FePt nanoparticles prepared by the chemical reduction method, Nano Lett, 3 (2003) 1647-1649.

[49] H. Zhang, D.R. Lin, G.T. Xu, J.B. Zheng, N.W. Zhang, Y.H. Li, B.H. Chen, Facile synthesis of carbon supported Pt-nanoparticles with Fe-rich surface: A highly active catalyst for preferential CO oxidation, Int J Hydrogen Energ, 40 (2015) 1742-1751.

[50] H.L. Liu, F. Nosheen, X. Wang, Noble metal alloy complex nanostructures: controllable synthesis and their electrochemical property, Chem Soc Rev, 44 (2015) 3056-3078.

[51] N. Wakabayashi, M. Takeichi, H. Uchida, M. Watanabe, Temperature dependence of oxygen reduction activity at Pt-Fe, Pt-Co, and Pt-Ni alloy electrodes, J Phys Chem B, 109 (2005) 58365841.

[52] Y.J. Xiong, J.Y. Chen, B. Wiley, Y.N. Xia, S. Aloni, Y.D. Yin, Understanding the role of oxidative etching in the polyol synthesis of Pd nanoparticles with uniform shape and size, J Am Chem Soc, 127 (2005) 7332-7333.

[53] H. Chen, Y.C. Yu, H.L.L. Xin, K.A. Newton, M.E. Holtz, D.L. Wang, D.A. Muller, H.D. Abruna, F.J. DiSalvo, Coalescence in the Thermal Annealing of Nanoparticles: An in Situ STEM Study of the Growth Mechanisms of Ordered Pt-Fe Nanoparticles in a KCl Matrix, Chem Mater, 25 (2013) 1436-1442.

[54] N. Worz, A. Brandner, P. Claus, Platinum-Bismuth-Catalyzed Oxidation of Glycerol: Kinetics and the Origin of Selective Deactivation, J Phys Chem C, 114 (2010) 1164-1172. [55] J.E.N. Brainer, D.C.S. Sales, E.B.M. Medeiros, N.M. Lima, C.A.M. Abreu, Wet Oxidation of Glycerol into Fine Organic Acids: Catalyst Selection and Kinetic Evaluation, Braz J Chem Eng, 31 (2014) 913-923.

[56] R. Alcala, M. Mavrikakis, J.A. Dumesic, DFT studies for cleavage of C-C and C-O bonds in surface species derived from ethanol on Pt(111), J Catal, 218 (2003) 178-190.

[57] A. Villa, G.M. Veith, L. Prati, Selective Oxidation of Glycerol under Acidic Conditions Using Gold Catalysts, Angew Chem Int Edit, 49 (2010) 4499-4502.

[58] S. Demirel, K. Lehnert, M. Lucas, P. Claus, Use of renewables for the production of chemicals: Glycerol oxidation over carbon supported gold catalysts, Appl Catal B-Environ, 70 (2007) 637-643. 